\title{
Discovery of ( \pm )-3-(1H-pyrazol-1-yl)-6,7-dihydro-5H-[1,2,4] triazolo[3,4-b][1,3,4] thiadiazine derivatives with promising in vitro anticoronavirus and antitumoral activity
}

\author{
Parameshwara Chary Jilloju ${ }^{1}$ Leentje Persoons ${ }^{2}$ - Sathish Kumar Kurapati ${ }^{3,4}$ - Dominique Schols ${ }^{2}$. \\ Steven De Jonghe ${ }^{2}$. Dirk Daelemans ${ }^{2} \cdot$ Rajeswar Rao Vedula $^{1}$ (D)
}

Received: 15 March 2021 / Accepted: 15 June 2021 / Published online: 24 June 2021

(C) The Author(s), under exclusive licence to Springer Nature Switzerland AG 2021

\begin{abstract}
A new series of ( \pm )-(3-(3,5-dimethyl-1H-pyrazol-1-yl)-6-phenyl-6,7-dihydro-5H-[1,2,4]triazolo[3,4-b][1,3,4]thiadiazin7-yl)(phenyl)methanones were efficiently synthesized starting from 4-amino-5-hydrazinyl-4H-1,2,4-triazole-3-thiol 1, acetyl acetone 2, various aromatic and heterocyclic aldehydes 3 and phenacyl bromides 4 . All the newly synthesized compounds were tested for their antiviral and antitumoral activity. It was shown that subtle structural variations on the phenyl moiety allowed to tune biological properties toward antiviral or antitumoral activity. Mode-of-action studies revealed that the antitumoral activity was due to inhibition of tubulin polymerization.
\end{abstract}

Graphic abstract

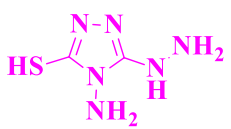

$0=$
$0=$

1

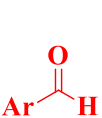

3
Anticorona virus activity

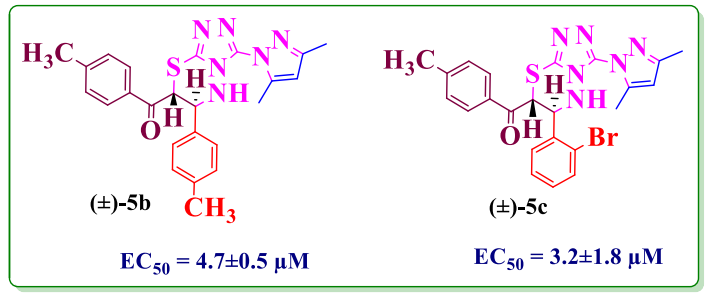<smiles>C1=CC=C1</smiles>

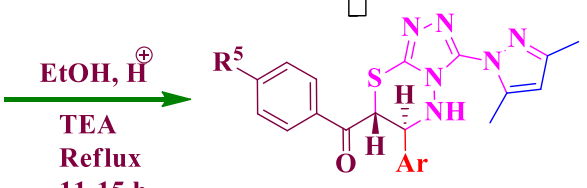

$( \pm)-5 a-t$

$83-94 \%$

Antitumoral activity

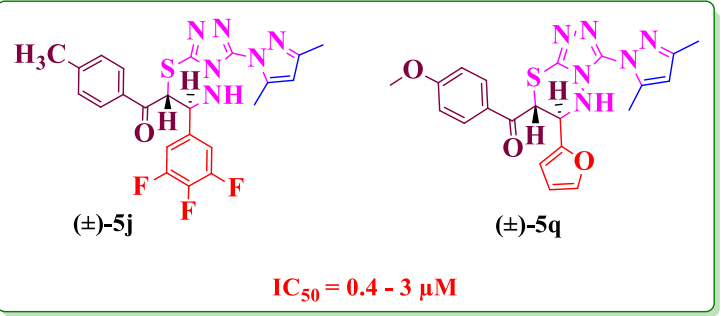

Extended author information available on the last page of the article 
Keywords Triazole $\cdot$ Pyrazole $\cdot$ Antiviral $\cdot$ Antitumoral $\cdot$ Dihydrothiadiazine $\cdot$ Multi-component reaction

\section{Introduction}

Heterocyclic structures are well-known components of various biologically active compounds. Nitrogen-containing hetero aromatics [1-7], such as triazole and pyrazole are well known to impart biological activity. Examples of marketed drugs based on a 1,2,4-triazole scaffold include voriconazole (an antifungal drug), forasartan (used for the treatment of hypertension), sitagliptin (an antidiabetic drug) and letrozole (a non-steroidal aromatase inhibitor for the treatment of breast cancer) (Fig. 1) [8]. In addition, a wide range of 1,2,4-triazole derivatives have been synthesized and tested in a wide variety of biological assays, leading to the discovery of anti-bacterial $[9,10]$, antiviral [11, 12], antifungal [13, 14], anti-inflammatory [15, 16], anti-proliferative [17, 18], anti-convulsant [19], anti-oxidant [20] and anti-Parkinson [21] triazole analogues. Pyrazole ring is another example of a hetero aromatic scaffold, exhibiting a wide range of biological properties. Examples of drugs based on a pyrazole scaffold that received marketing include celecoxib and deracoxib (both cyclo-oxygenase-2 inhibitors), surinabant (a cannabinoid receptor type 1 antagonist) and crizotinib (an ALK inhibitor). However, a plethora of other activities, such as anti-HIV [22, 23], anti-malarial [24], anti-oxidant [25], anti-inflammatory [26], anti-bacterial [27, 28], anti-tumor [29], anti-pyretic [30], anti-analgesic [31], anti-cancer [32] and anti-leishmanial [33] activities have been associated with the pyrazole scaffold.

Although sulfur-containing heterocyclic compounds were found to have extensive biological applications, 1,3,4-thiadiazines are explored to a much lesser extent in medicinal chemistry, when compared to 1,2,4-triazole and pyrazole motifs. Thiadiazines are themselves showing good biological activities [34-39].

Multi-component reactions (MCRs), also known as multicomponent assembly processes (MCAPs), are attractive synthetic methodologies in medicinal chemistry. The synthetic procedures in MCRs use mild reaction conditions and all, or most, of the atoms from the various reactants contribute to formation of the target compounds. The main advantages of MCRs are their atom economy, eco-friendliness and the fact that it allows to quickly generate structural diversity [40-44].

We recently reported the synthesis of $[1,2,4]$ triazolo[3,4$b][1,3,4]$ thiadiazines through the multi-component reaction (MCR) process [45]. The presence of a hydrazino group in these molecules offers the possibility to convert them into pyrazole moieties. In view of the numerous biological applications of triazoles, pyrazoles and thiadiazines we became interested in the synthesis of the title compounds. Final compounds were subjected to a variety of assays in order to find antiviral and/or antitumoral activity.

\section{Results and discussions}

The synthesis of the ( \pm )-3-(1H-pyrazol-1-yl)-6,7-dihydro$5 H$-[1,2,4] triazolo[3,4- $b][1,3,4]$ thiadiazine derivatives was performed using a two-step, one pot procedure. In order to optimize the chemistry, a model reaction was carried out using 4-amino-3-hydrazino-5-mercapto-1,2,4-triazole 1, acetylacetone 2, 2,3-dimethoxybenzaldehyde 3 and 4-methoxyphenacylbromide 4 as starting materials (Scheme 1).

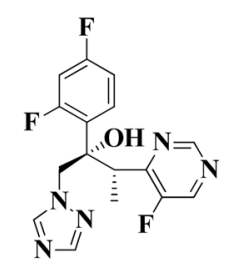

Voriconazole

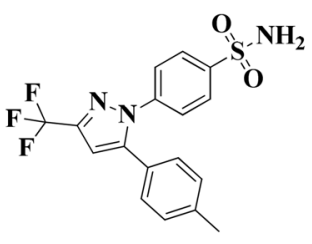

Celecoxib

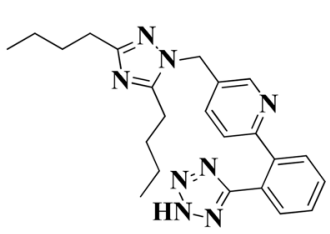

Forasartan

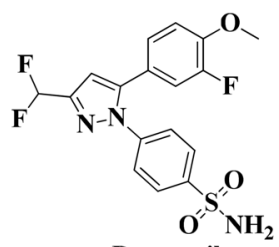

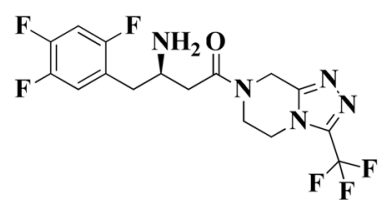

Sitagliptin<smiles>CCc1c(C(=O)NN2CCCCC2)nn(-c2ccc(Cl)cc2Cl)c1-c1ccc(Br)cc1</smiles>

Surinabant<smiles>N#Cc1ccc(C(c2ccc(C#N)cc2)(c2cccnc2)c2ccnnn2)cc1</smiles>

Letrozole

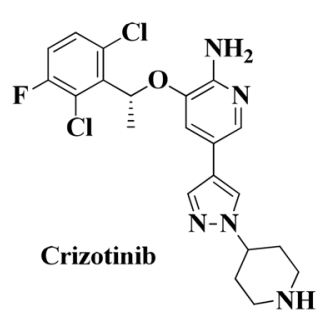

Fig. 1 Marketed drugs based on a 1,2,4-triazole and pyrazole scaffold 


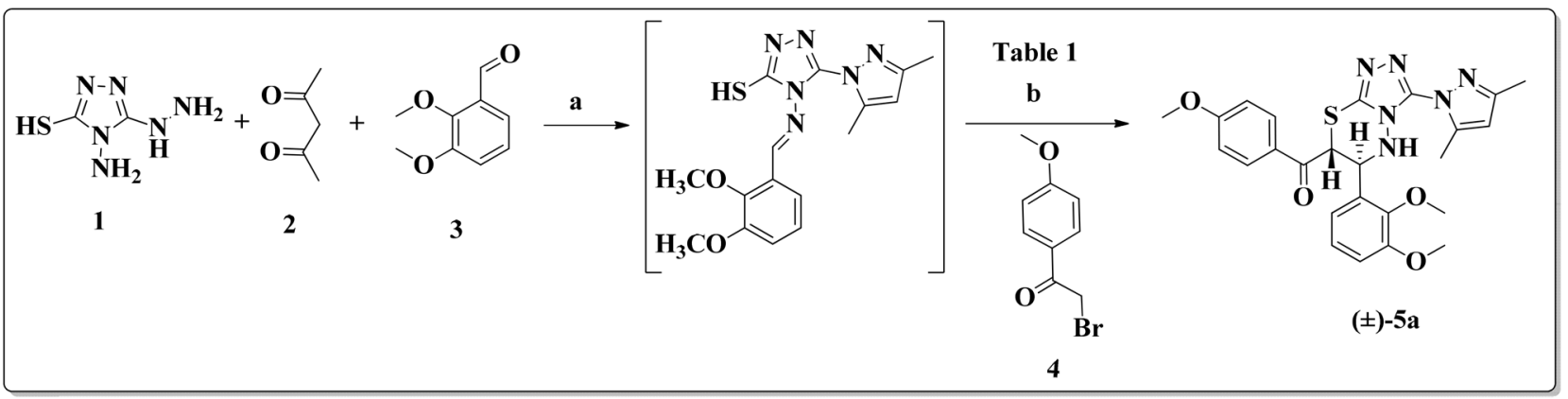

Scheme 1 Model reaction. Reaction conditions: a 1 (1 mmol), 2 (1 mmol), 3 (1 mmol), EtOH, $\mathrm{HCl}$ (one drop), b 4 (1 mmol), TEA (3 mmol), $\mathrm{EtOH}$, reflux

Table 1 Screening of the base catalyst

\begin{tabular}{llllll}
\hline Entry & Solvent & Base & Temp. $\left({ }^{\circ} \mathrm{C}\right)$ & Time $(\mathrm{h})$ & $\begin{array}{l}\text { Yield }(\%) \\
\text { of }( \pm)-5 \mathrm{a}\end{array}$ \\
\hline 1 & EtOH & - & 70 & 10 & 0 \\
2 & EtOH & Pyridine & 70 & 16 & 55 \\
3 & EtOH & Piperidine & 70 & 12 & 48 \\
4 & EtOH & Triethylamine & 70 & 11.30 & 92 \\
\hline
\end{tabular}

The first step of the reaction was carried out in ethanol as solvent at reflux temperature, in the presence of a catalytic amount of $\mathrm{HCl}$ yielding the intermediate 5-(3,5-dimethyl-1 $H$-pyrazol-1-yl)-4-((4-methoxybenzylidene)
amino)-4H-1,2,4-triazole-3-thiol [46]. The intermediate was not isolated instead of it 4-methoxyphenacylbromide 4 was added to the reaction mixture. In order to drive the ring closure to form the thiadiazine moiety, various reaction conditions were explored (Table 1). Running this reaction, either at room temperature or at reflux temperature failed to yield the desired product. Upon the addition of an organic base (such as Pyridine, Piperidine or Triethylamine), the desired product was formed. Using triethylamine as base and running the reaction at reflux temperature (entry 4) resulted in the formation of desired compound $( \pm)-5 \mathrm{a}$ in excellent yield (Table 1).

Using this methodology (Scheme 2), a series of compounds was prepared using various benzaldehydes,

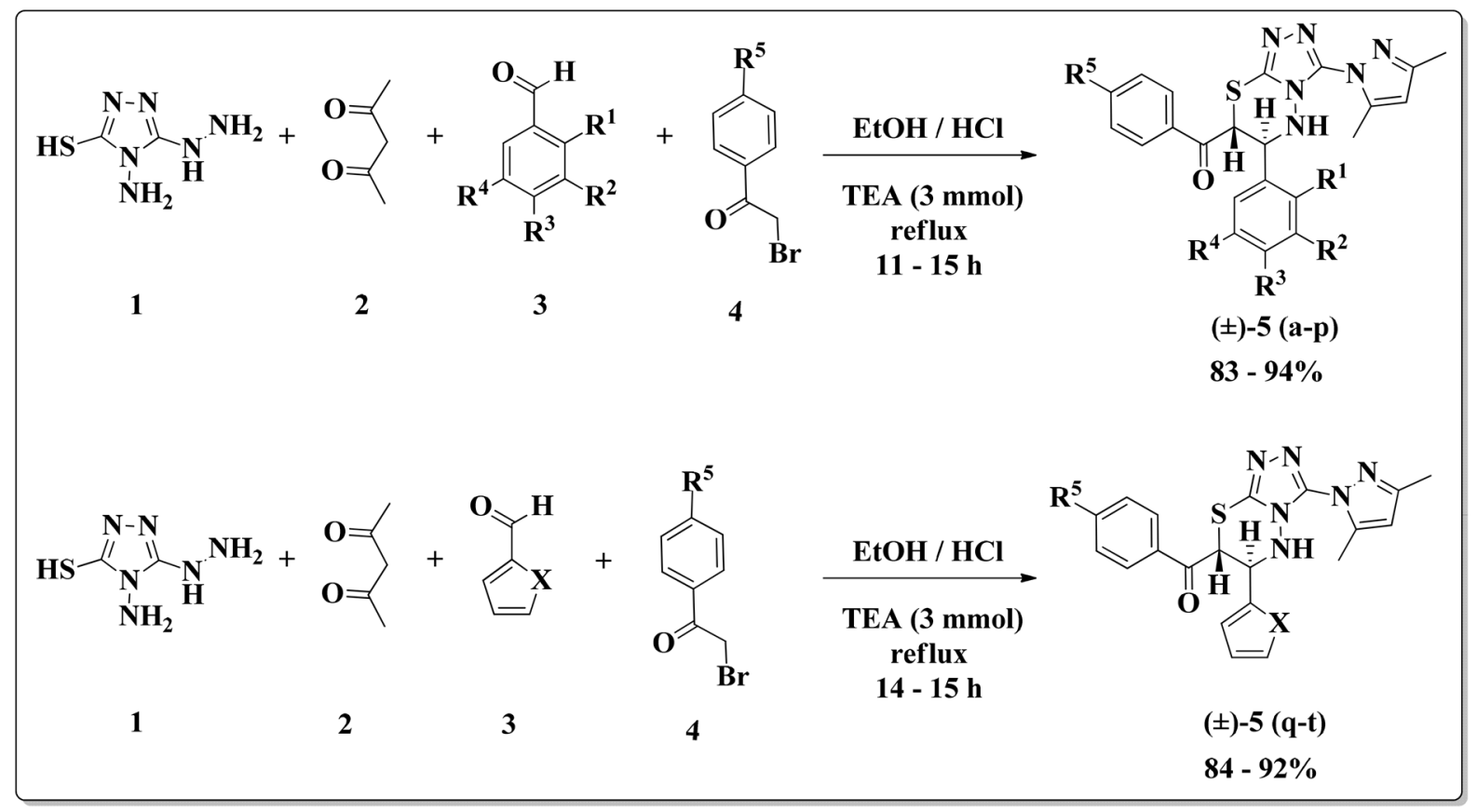

Scheme 2 One-pot, four-component synthesis of ( \pm )-3-(1H-pyrazol-1-yl)-6,7-dihydro-5H-[1,2,4]triazolo[3,4- $b][1,3,4]$ thiadiazine derivatives (5a-t) 
heterocyclic aldehydes and phenacyl bromides (Table 2). This approach is simple and affords the desired products in yields ranging from 83 to $94 \%$. (Table 3 ).

In the present investigation, pyrazole and dihydrothiadiazine skeletons were developed using one-pot, four-component reaction. Initially, hydrazino functional group of compound 1 underwent cyclocondensation with acetylacetone 2 to form pyrazole ring [47]. Then an appropriate amount of different aldehydes 3 and substituted phenacyl bromides 4 were reacted with amine $\left(-\mathrm{NH}_{2}\right)$ and thiol $(-\mathrm{SH})$ groups of compound 1 respectively by using triethylamine to establish the dihydrothiadiazines (Scheme 3) [48].

The structures of the final products were confirmed by their spectral data. The FT-IR spectrum of product $( \pm)-5 b$ showed a characteristic stretching band at $1681 \mathrm{~cm}^{-1}$ corresponding to the $-\mathrm{C}=\mathrm{O}$ functional group, whereas the -NH- group appeared at $3135 \mathrm{~cm}^{-1}$. The ${ }^{1} \mathrm{H}-\mathrm{NMR}$ spectrum of compound ( \pm )-5b showed characteristic peaks, such as two singlets at 2.21 and $2.95 \mathrm{ppm}$, arising from the two methyl groups on the pyrazole ring. Another two singlets appeared at 2.37 and $2.43 \mathrm{ppm}$ that were assigned to the methyl groups on both phenyl moieties. The two $-\mathrm{CH}$ - protons of the dihydrothiadiazine skeleton were visible as two doublets at 5.05 and 5.25 ppm, respectively. The proton of the pyrazole ring showed up as a singlet at $6.00 \mathrm{ppm}$, whereas the $-\mathrm{NH}-$ proton appeared at $7.42 \mathrm{ppm}$. The remaining aromatic protons appeared in the region of 7.11-7.80 ppm. The ${ }^{13} \mathrm{C}-\mathrm{NMR}$ spectrum of compound $( \pm)-5 \mathrm{~b}$ showed peaks at 11.9 and $13.6 \mathrm{ppm}$ for the carbon
Table 3 Hydrogen bonding interactions

\begin{tabular}{lllll}
\hline S. no & D-H...A & H...A $(\AA)$ & D...A $(\AA)$ & D-H...A $\left(^{\circ}\right)$ \\
\hline 1 & $\mathrm{~N}(6)-\mathrm{H}(6) \ldots \mathrm{N}(2)^{\mathrm{i}}$ & 2.50 & 3.1298 & 130 \\
2 & $\mathrm{C}(4)-\mathrm{H}(4) \ldots \mathrm{N}(4)^{\mathrm{i}}$ & 2.38 & 3.2637 & 150 \\
3 & $\mathrm{C}(19)-\mathrm{H}(19) \ldots \mathrm{O}(1)^{\mathrm{ii}}$ & 2.46 & 3.3727 & 160 \\
\hline
\end{tabular}

Symmetry transformations used: (i) $1 / 2-x, 1 / 2+y, 1 / 2+z$; (ii) $-1 / 2-x$, $1 / 2+y, 1 / 2-z$

atoms of two methyl groups on the pyrazole ring at 21.1 and $21.8 \mathrm{ppm}$ for the carbons of two methyl groups on the phenyl moiety. The characteristic carbons of the dihydrothiadiazine skeleton appeared at 44.2 and $59.3 \mathrm{ppm}$ respectively. The pyrazole carbon displayed a peak at $107.8 \mathrm{ppm}$, whereas the carbonyl peak appeared as the most downfield signal at $193.7 \mathrm{ppm}$. The remaining aromatic carbons appeared in the range of 127.3 to $151.8 \mathrm{ppm}$. Mass spectral analysis of compound ( \pm )-5b showed a molecular ion peak at $m / z 445$.

\section{X-ray crystallography}

To confirm the structure, crystalline material of compound ( \pm )-5 $\mathrm{h}$ was isolated, and single crystal X-ray diffraction data were obtained. The compound crystallizes in a monoclinic $P 2_{1} / n$ space group. The molecular structure of $( \pm)-5 \mathrm{~h}$ in ORTEP representation is shown in Fig. 2.

Compound $( \pm)-5 \mathrm{~h}$ has a 4-methylbenzoyl group and 4-chlorophenyl group on two adjacent chiral centers of the
Table 2 Derivatives of (土)-3-(1H-pyrazol-1-yl)6,7-dihydro- $5 H$-[1,2,4] triazolo $[3,4-b][1,3,4]$ thiadiazine $(5 \mathrm{a}-\mathrm{t})$

\begin{tabular}{lllllllll}
\hline Product & $\mathrm{R}^{1}$ & $\mathrm{R}^{2}$ & $\mathrm{R}^{3}$ & $\mathrm{R}^{4}$ & $\mathrm{R}^{5}$ & $\mathrm{X}$ & Time (h) & Yield (\%) \\
\hline $5 \mathrm{a}$ & $\mathrm{OCH}_{3}$ & $\mathrm{OCH}_{3}$ & $\mathrm{H}$ & $\mathrm{H}$ & $\mathrm{OCH}_{3}$ & - & 11.30 & 92 \\
$5 \mathrm{~b}$ & $\mathrm{H}$ & $\mathrm{H}$ & $\mathrm{CH}_{3}$ & $\mathrm{H}$ & $\mathrm{CH}_{3}$ & - & 11.00 & 91 \\
$5 \mathrm{c}$ & $\mathrm{H}$ & $\mathrm{H}$ & $\mathrm{NO}_{2}$ & $\mathrm{H}$ & $\mathrm{NO}_{2}$ & - & 14.30 & 83 \\
$5 \mathrm{~d}$ & $\mathrm{H}$ & $\mathrm{OCH}_{3}$ & $\mathrm{H}$ & $\mathrm{H}$ & $\mathrm{Cl}$ & - & 12.00 & 86 \\
$5 \mathrm{e}$ & $\mathrm{Br}$ & $\mathrm{H}$ & $\mathrm{H}$ & $\mathrm{H}$ & $\mathrm{F}$ & - & 12.30 & 90 \\
$5 \mathrm{f}$ & $\mathrm{Br}$ & $\mathrm{H}$ & $\mathrm{H}$ & $\mathrm{H}$ & $\mathrm{CH}_{3}$ & - & 11.50 & 92 \\
$5 \mathrm{~g}$ & $\mathrm{H}$ & $\mathrm{H}$ & $\mathrm{Cl}$ & $\mathrm{H}$ & $\mathrm{Br}$ & - & 13.00 & 90 \\
$5 \mathrm{~h}$ & $\mathrm{H}$ & $\mathrm{H}$ & $\mathrm{Cl}$ & $\mathrm{H}$ & $\mathrm{CH}_{3}$ & - & 12.00 & 93 \\
$5 \mathrm{i}$ & $\mathrm{H}$ & $\mathrm{OCH}$ & $\mathrm{OH}$ & $\mathrm{OCH}$ & $\mathrm{H}$ & - & 11.40 & 89 \\
$5 \mathrm{j}$ & $\mathrm{H}$ & $\mathrm{F}$ & $\mathrm{F}$ & $\mathrm{F}$ & $\mathrm{CH}_{3}$ & - & 14.00 & 92 \\
$5 \mathrm{k}$ & $\mathrm{H}$ & $\mathrm{F}$ & $\mathrm{F}$ & $\mathrm{F}$ & $\mathrm{H}_{3}$ & - & 13.30 & 90 \\
$5 \mathrm{l}$ & $\mathrm{H}$ & $\mathrm{OCH}$ & $\mathrm{OCH}_{3}$ & $\mathrm{OCH}_{3}$ & $\mathrm{~F}_{3}$ & - & 13.15 & 88 \\
$5 \mathrm{~m}$ & $\mathrm{H}$ & $\mathrm{OCH}$ & $\mathrm{OCH}_{3}$ & $\mathrm{OCH}_{3}$ & $\mathrm{H}$ & - & 11.40 & 94 \\
$5 \mathrm{n}$ & $\mathrm{Cl}$ & $\mathrm{H}$ & $\mathrm{H}$ & $\mathrm{H}$ & $\mathrm{OCH}_{3}$ & - & 14.15 & 86 \\
$5 \mathrm{o}$ & $\mathrm{OCH}$ & $\mathrm{H}$ & $\mathrm{OCH}_{3}$ & $\mathrm{H}$ & $\mathrm{OCH}_{3}$ & - & 12.00 & 94 \\
$5 \mathrm{p}$ & $\mathrm{OCH}$ & $\mathrm{H}$ & $\mathrm{OCH}_{3}$ & $\mathrm{H}$ & $\mathrm{NO}_{2}$ & - & 14.00 & 92 \\
$5 \mathrm{q}$ & - & - & - & - & $\mathrm{OCH}_{3}$ & $\mathrm{O}$ & 14.30 & 87 \\
$5 \mathrm{r}$ & - & - & - & - & $\mathrm{CH}_{3}$ & $\mathrm{O}$ & 14.15 & 85 \\
$5 \mathrm{~s}$ & - & - & - & - & $\mathrm{NO}_{2}$ & $\mathrm{~S}$ & 15.00 & 89 \\
$5 \mathrm{t}$ & - & - & - & - & $\mathrm{NO}_{2}$ & $\mathrm{O}$ & 14.50 & 92 \\
\hline & & & & & & & & \\
\hline
\end{tabular}




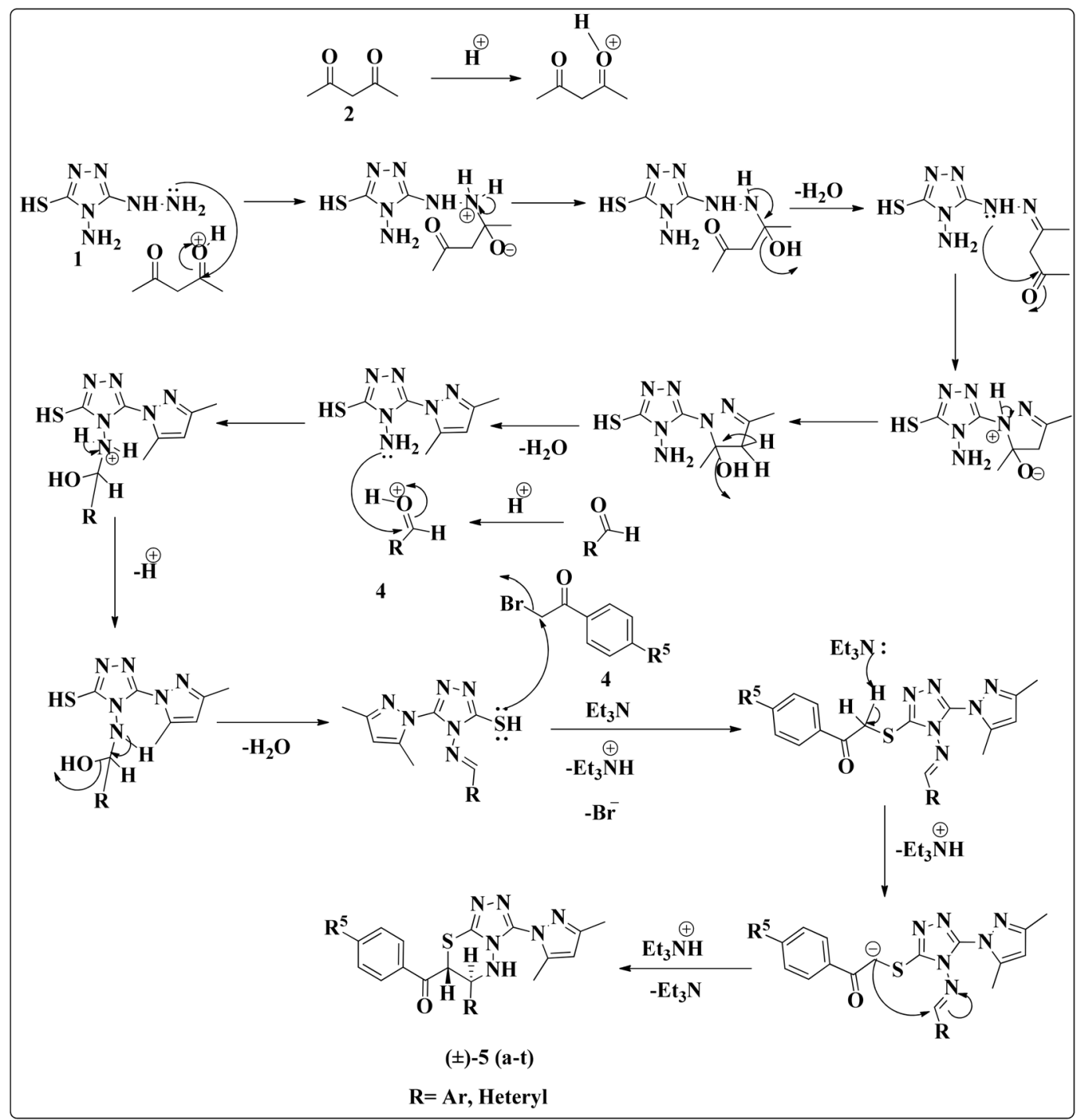

Scheme 3 Plausible mechanism for the synthesis of compounds ( \pm )-5a-t

six-membered dihydrothiadiazine ring. The dihydrothiadiazine moiety is fused with a triazole ring, further connected to a pyrazole ring through a carbon-nitrogen single bond. The phenyl rings of 4-methylbenzoyl and 4-chlorophenyl groups are almost perpendicular $\left(79.92^{\circ}\right.$ and $82.28^{\circ}$ respectively) to the mean plane of the fused six- and five-membered rings. The pyrazole ring attached to triazole makes an angle of $55.59^{\circ}$ with the mean plane of the fused six- and five-membered rings. The bond distances and angles are consistent with the structure derived from NMR data. The centrosymmetric space group $\left(P 2_{1} / n\right)$ indicates (Table 4$)$ that the material is a racemic mixture. The unit cell contains two pairs of enantiomers and is connected through non-covalent interactions.

Non-covalent intermolecular interactions, such as hydrogen bonding, play an essential role in binding of drugs to their targets, such as DNA or proteins. In this context, the possibility of the presence of non-covalent interactions in the solid state structure of compound $( \pm)-5 \mathrm{~h}$ was explored. As a result, we were able to identify one $\mathrm{N}-\mathrm{H}$... N hydrogen bonding, one $\mathrm{C}-\mathrm{H} \ldots \mathrm{O}$ interaction and one $\mathrm{C}-\mathrm{H} \ldots$ $\mathrm{N}$ interaction (Fig. 3). The interactions and corresponding symmetry transformations are listed in Table 3 .

\section{Biological evaluation}

\section{In vitro antiviral screening}

Compounds $(( \pm)-5 \mathrm{a}-\mathrm{t})$ were subjected to a broad antiviral screening. At a concentration of $100 \mu \mathrm{M}$, no selective antiviral activity was observed for the following viruses: influenza A (H1N1 and H3N2) and influenza B virus (in 
Fig. 2 ORTEP representation of compound $( \pm)-5 \mathrm{~h}$

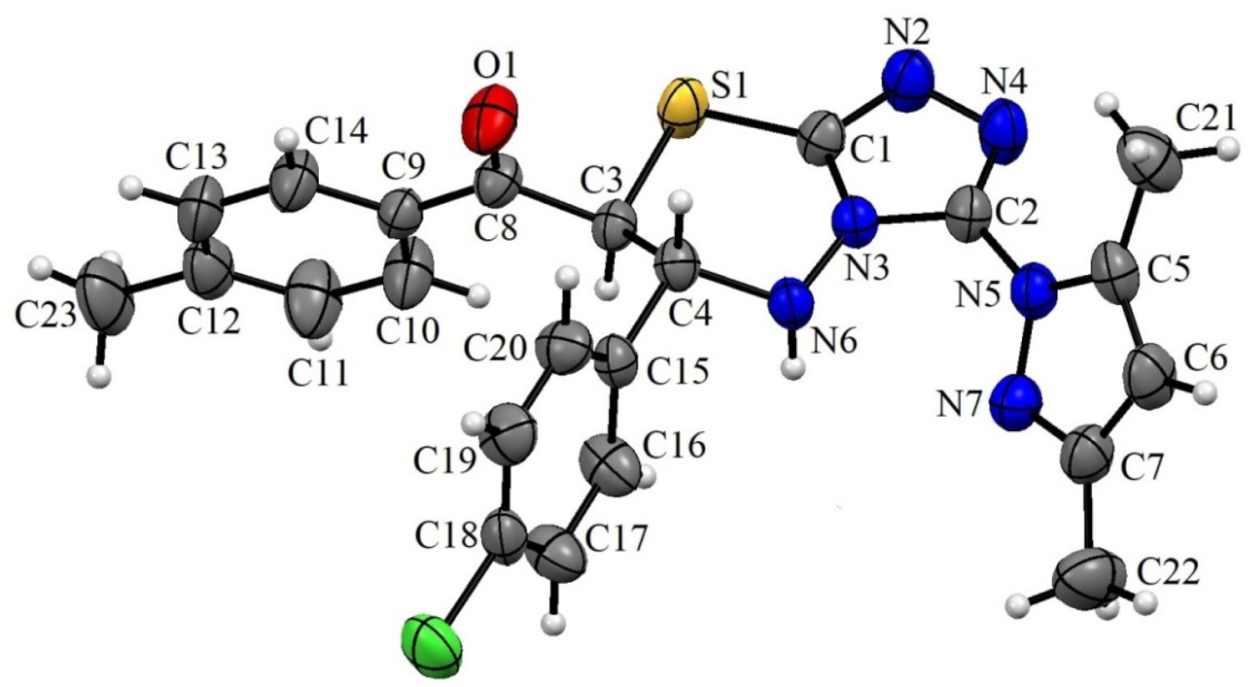

Table 4 Important crystallographic data for compound ( \pm ) $-5 \mathrm{~h}$

\begin{tabular}{|c|c|}
\hline Compound & $( \pm)-5 \mathrm{~h}$ \\
\hline Chemical formula & $\mathrm{C}_{23} \mathrm{H}_{21} \mathrm{ClN}_{6} \mathrm{OS}$ \\
\hline Formula weight & 464.97 \\
\hline Crystal system & Monoclinic \\
\hline Space group & $P 2_{1} / \mathrm{n}$ \\
\hline$a(\AA)$ & $14.2063(18)$ \\
\hline$b(\AA)$ & $8.4877(11)$ \\
\hline$c(\AA)$ & $20.022(3)$ \\
\hline$\alpha\left(^{\circ}\right)$ & 90 \\
\hline$\beta\left({ }^{\mathrm{o}}\right)$ & $107.576(5)$ \\
\hline$\gamma\left({ }^{\circ}\right)$ & 90 \\
\hline$V\left(\AA^{3}\right)$ & $2301.6(5)$ \\
\hline$Z$ & 4 \\
\hline$\rho\left(\mathrm{g} \mathrm{cm}^{-3}\right)$ & 1.342 \\
\hline$\mu\left(\mathrm{mm}^{-1}\right)$ & 0.285 \\
\hline Reflections collected & 34,696 \\
\hline Reflections unique & 4077 \\
\hline Reflections $[I \geq 2 \sigma(I)]$ & 4077 \\
\hline Parameters & 289 \\
\hline$R 1, w R 2[I \geq 2 \sigma(I)]$ & $0.0471,0.1330$ \\
\hline$R 1, w R 2$ [all data] & $0.0537,0.1387$ \\
\hline GOF on $F^{2}$ & 1.136 \\
\hline Max./Min. $\Delta \rho\left(\mathrm{e}^{-3}\right)$ & -0.710 \\
\hline
\end{tabular}

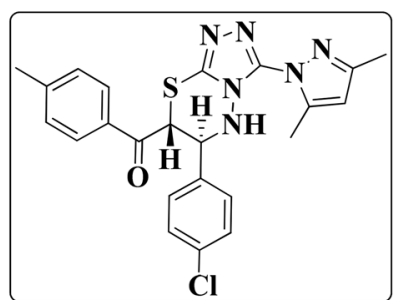

MDCK cells), respiratory syncytial virus (in HEp-2cells), yellow fever virus (in Huh7 cells), herpes simplex virus type 1 and 2 (in HEL 299 cells). However, a number of derivatives did show antiviral activity against the human corona virus 229E (hCoV-229E) in HEL 299 cells (Table 5). Especially compounds $( \pm)-5 \mathrm{~b}$ and $( \pm)-5 \mathrm{f}$ displayed promising activity with $\mathrm{EC}_{50}$ values of 4.7 and $3.2 \mu \mathrm{M}$, respectively. In addition, both derivatives lacked cytotoxicity for the HEL cells giving rise to favorable selectivity indexes.

\section{In vitro antitumoral screening}

To investigate their anti-cancer potential, compounds 5a-t were tested in vitro for their anti-proliferative properties, using a real-time IncuCyteproliferation assay against an array of solid and hematological cancers including LN-229 (glioblastoma), Capan-1 (pancreatic adenocarcinoma), HCT116 (colorectal carcinoma), NCI-H460 (lung carcinoma), DND-41 (acute lymphoblastic leukemia), HL-60 (acute myeloid leukemia), K-562 (chronic myeloid leukemia) and Z-138 (non-Hodgkin lymphoma) cell lines. Docetaxel (a microtubule depolymerisation inhibitor) and staurosporine (STS, a pan-kinase inhibitor) were used as positive controls. From this screening campaign, two derivatives (compounds $5 \mathrm{j}$ and $5 \mathrm{q}$ ) emerged that showed low $\mu \mathrm{M}$ activity against the different cell lines (Table 6). 


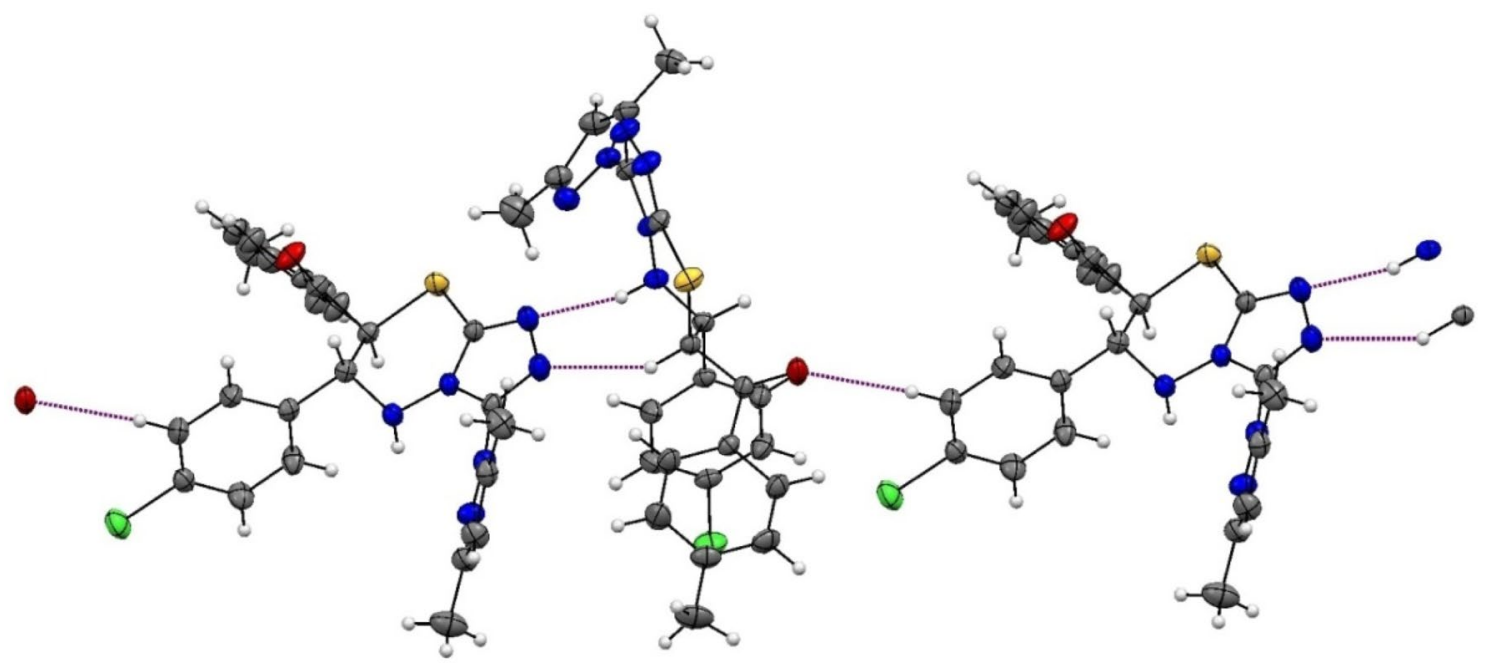

Fig. 3 Intermolecular Hydrogen bonding interactions of compound $( \pm)-5 \mathrm{~h}$ in crystal lattice

Table 5 Antiviral evaluation of compounds $( \pm)-5 \mathrm{a}-\mathrm{t}$ against $\mathrm{hCoV}-$ $229 \mathrm{E}$

\begin{tabular}{llll}
\hline Compound & Conc. unit & \multicolumn{2}{c}{ hCoV-229E (HEL cells) } \\
\cline { 2 - 4 } & & $\mathrm{CC}_{50}$ & $\mathrm{EC}_{50}$ \\
\hline $5 \mathrm{~b}$ & $\mu \mathrm{M}$ & 81.5 & $4.7 \pm 0.5\left(^{*}\right)$ \\
$5 \mathrm{c}$ & $\mu \mathrm{M}$ & $>100$ & 24.4 \\
$5 \mathrm{e}$ & $\mu \mathrm{M}$ & $>100$ & $>100$ \\
$5 \mathrm{f}$ & $\mu \mathrm{M}$ & $>100$ & $3.2 \pm 1.8\left(^{*}\right)$ \\
$5 \mathrm{~g}$ & $\mu \mathrm{M}$ & $>100$ & $>100$ \\
$5 \mathrm{~h}$ & $\mu \mathrm{M}$ & $>100$ & 38.0 \\
$5 \mathrm{j}$ & $\mu \mathrm{M}$ & 23.8 & $>100$ \\
$5 \mathrm{k}$ & $\mu \mathrm{M}$ & $>100$ & $>100$ \\
$5 \mathrm{~m}$ & $\mu \mathrm{M}$ & $>100$ & 95.7 \\
$5 \mathrm{n}$ & $\mu \mathrm{M}$ & $>100$ & $>100$ \\
$5 \mathrm{p}$ & $\mu \mathrm{M}$ & $>100$ & $>100$ \\
$5 \mathrm{q}$ & $\mu \mathrm{M}$ & $<0.8$ & $>100$ \\
$5 \mathrm{r}$ & $\mu \mathrm{M}$ & $>100$ & $>100$ \\
$5 \mathrm{~s}$ & $\mu \mathrm{M}$ & $>100$ & $>100$ \\
$5 \mathrm{t}$ & $\mu \mathrm{M}$ & $>100$ & $>100$ \\
$\mathrm{UDA}$ & $\mu \mathrm{g} / \mathrm{ml}$ & $>100$ & 2.1 \\
\hline
\end{tabular}

(*)Mean value of three independent experiments \pm SEM

Because of the promising antitumoral profile of compounds $( \pm)-5 \mathrm{j}$ and $( \pm)-5 \mathrm{q}$, their apoptogenic potential in non-cancerous peripheral blood mononuclear cells (PBMCs) was determined as counter screening. The activation of the executioner caspases- 3 and -7 normally precedes the manifestation of apoptosis as massive DNA fragmentation. Therefore, the caspase-3/7 Green reagent was added to the PBMCs, which are also treated with different concentrations of compounds $( \pm)-5 \mathrm{j}$ and $( \pm)-5 \mathrm{q}$. When activated caspase 3 or 7 are intracellularly present, they will cleave the
Caspase-3/7 Green Reagent at the DEVD motif. This results in the release of a DNA binding dye that fluorescently labels nuclear DNA of apoptic cells. In addition, in order to distinguish dead cells from live cells, a propidium iodide (PI) staining was carried out. As can be derived from Fig. 4, only very high concentrations of compounds $( \pm)-5 j$ and $( \pm)-5 q$ $(100 \mu \mathrm{M})$ give rise to a small increase in the number of apoptotic and dead cells. Overall, these data indicate that compounds $( \pm)-5 \mathrm{j}$ and $( \pm)-5 \mathrm{q}$ did not inhibit the viability of normal PBMCs and demonstrate selectivity toward cancer cells over normal cells (Fig. 4).

Despite their promising antitumoral profile, the exact molecular target of compounds ( \pm ) $-5 \mathrm{j}$ and $( \pm)-5 \mathrm{q}$ remained elusive. In order to assess whether they interact with tubulin, an immune fluorescence analysis of tubulin in HEp-2 cells treated for $3 \mathrm{~h}$ with compounds $( \pm)-5 \mathrm{j}$ and $( \pm)-5 \mathrm{q}$ was performed, and compared to DMSO (vehicle control) and to vincristine (a known tubulin polymerization inhibitor, used as positive control). It can be clearly observed that both compounds $( \pm)-5 \mathrm{j}$ and $( \pm)-5 \mathrm{q}$ inhibit the polymerization of tubulin in a dose-dependent manner (Fig. 5).

\section{Conclusion}

The synthesis of a new series of ( \pm )-3-(1H-pyrazol-1-yl)6,7-dihydro-5 $H$ - $[1,2,4]$ triazolo[3,4- $b][1,3,4]$ thiadiazine derivatives was carried out in an excellent yields via a onepot, four-component method using readily available starting materials. The reactions proceeds in such a way with high atom economy, leading to the formation of one $\mathrm{C}=\mathrm{N}$, two $\mathrm{C}-\mathrm{N}$, one $\mathrm{C}-\mathrm{C}$, and one $\mathrm{C}-\mathrm{S}$ bonds in a single operation, giving multi-annulated products. All the final compounds were tested for their antiviral and antitumoral activity. It 
Table 6 Antitumoral evaluation of compounds from ( \pm ) $-5 \mathrm{a}-\mathrm{t} . \mathrm{IC}_{50}$

\begin{tabular}{|c|c|c|c|c|c|c|c|c|}
\hline \multirow[t]{2}{*}{ Compound } & \multicolumn{8}{|l|}{$\mathrm{IC}_{50}(\mu \mathrm{M})$} \\
\hline & LN-229 & Capan-1 & HCT-116 & NCI-H460 & DND-41 & HL-60 & K-562 & Z-138 \\
\hline $5 b$ & 47.1 & 57.5 & 67.8 & $>100$ & 39.3 & 50.9 & 10.4 & 48.4 \\
\hline $5 \mathrm{c}$ & $>100$ & $>100$ & $>100$ & $>100$ & $>100$ & $>100$ & $>100$ & $>100$ \\
\hline $5 \mathrm{e}$ & $>100$ & $>100$ & $>100$ & $>100$ & $>100$ & $>100$ & $>100$ & $>100$ \\
\hline $5 f$ & $>100$ & $>100$ & $>100$ & $>100$ & $>100$ & $>100$ & $>100$ & $>100$ \\
\hline $5 \mathrm{~g}$ & $>100$ & $>100$ & $>100$ & $>100$ & $>100$ & $>100$ & $>100$ & $>100$ \\
\hline $5 \mathrm{~h}$ & $>100$ & $>100$ & $>100$ & $>100$ & $>100$ & $>100$ & $>100$ & $>100$ \\
\hline $5 \mathrm{j}^{*}$ & $2.7 \pm 0.2$ & $2.3 \pm 0.2$ & $2.5 \pm 0.09$ & 56.0 & $2.4 \pm 0.4$ & $13.0 \pm 2.8$ & $3.4 \pm 0.2$ & $1.9 \pm 0.03$ \\
\hline $5 \mathrm{k}$ & $>100$ & $>100$ & $>100$ & $>100$ & $>100$ & $>100$ & $>100$ & $>100$ \\
\hline $5 \mathrm{~m}$ & $>100$ & $>100$ & $>100$ & $>100$ & $>100$ & $>100$ & $>100$ & $>100$ \\
\hline $5 n$ & 68.3 & 63.1 & $>100$ & $>100$ & 91.7 & 71.2 & 53.2 & 53.9 \\
\hline $5 p$ & $>100$ & $>100$ & $>100$ & $>100$ & $>100$ & $>100$ & $>100$ & $>100$ \\
\hline $5 q^{*}$ & $0.7 \pm 0.09$ & $1.1 \pm 0.7$ & $1.0 \pm 0.4$ & $2.5 \pm 0.2$ & $0.6 \pm 0.2$ & $2.0 \pm 0.4$ & $2.3 \pm 1.6$ & $0.4 \pm 0.005$ \\
\hline $5 \mathrm{r}$ & 43.9 & 54.3 & 69.0 & 47.0 & 70.2 & 54.0 & 23.5 & 50.2 \\
\hline $5 \mathrm{~s}$ & $>100$ & $>100$ & $>100$ & $>100$ & $>100$ & $>100$ & $>100$ & $>100$ \\
\hline $5 \mathrm{t}$ & 62.7 & $>100$ & $>100$ & $>100$ & $>100$ & 74.5 & 79.3 & 49.5 \\
\hline Docetaxel* & $0.0087 \pm 0.0004$ & $0.0042 \pm 0.0021$ & $0.0009 \pm 0.0008$ & $0.0038 \pm 0.0029$ & $0.0033 \pm 0.0014$ & $0.0023 \pm 0.0003$ & $0.0037 \pm 0.0003$ & $0.0011 \pm 0.0008$ \\
\hline STS* & $0.0229 \pm 0.0021$ & $0.0007 \pm 0.0002$ & $0.0004 \pm 0.0001$ & $0.00010 \pm 0.0000$ & $0.0015 \pm 0.0004$ & $0.0043 \pm 0.0022$ & $0.0074 \pm 0.0017$ & $0.0224 \pm 0.0074$ \\
\hline
\end{tabular}

*Mean value of two independent experiments \pm SEM
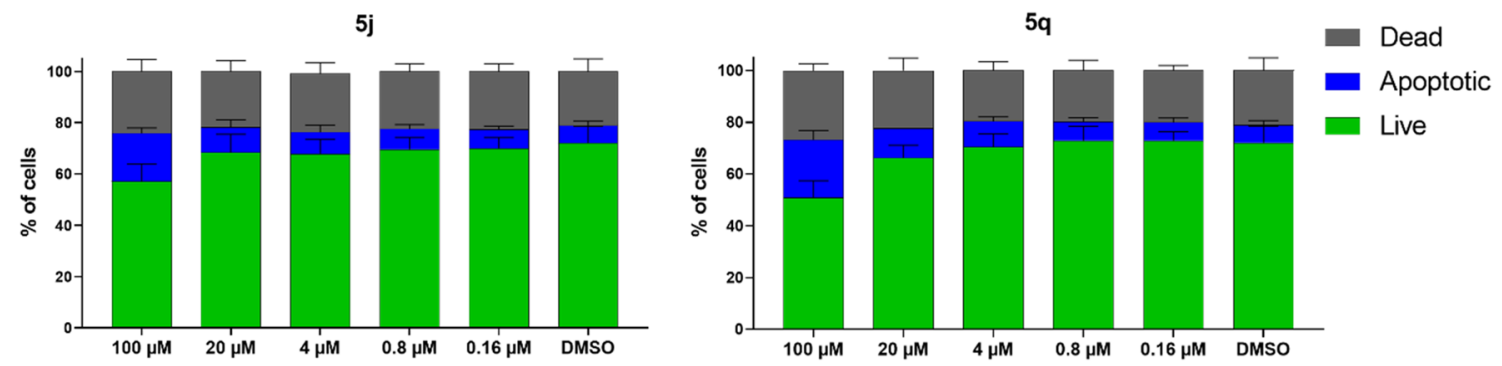

Fig. 4 Analysis of apoptosis induction by compound-5j (left) and ( \pm )-5q (right) in PBMC originating from two healthy donors

was demonstrated that subtle structural modifications on the phenyl moieties allowed to tune the biological properties of the compounds. Among the newly synthesized compounds, a number of derivatives show promising antiviral activity against the hCoV-229E, whereas other derivatives exhibited cytotoxicity in various cancer cell lines. In addition, it was demonstrated that the antitumoral activity of these compounds is caused by inhibition of tubulin polymerization.

\section{Experimental}

\section{General}

All the reactants, reagents and solvents were pure, purchased from commercial sources and used without further purification. All the synthesized compounds were preliminarily confirmed by monitoring using TLC plates (E, Merck, Mumbai, India) in the UV-light chamber. A "Stuart SMP30" programmable melting point instrument (Bibby Scientific Ltd. U.K.) was used to record the melting points of the synthesized compounds. FT-IR spectra of the newly synthesized compounds in $\mathrm{KBr}$-pellets were recorded on a PerkinElmer 100S FT-IR spectrophotometer. The ${ }^{1} \mathrm{H}$ - and the ${ }^{13} \mathrm{C}$-NMR chemical shift values were determined for the compounds on Avance-III Bruker WM-400 MHz spectrometer in $\delta$ ppm. Tetramethylsilane (TMS) acts as reference standard for the chemical shifts. Suitable deuterated solvents like $\mathrm{CDCl}_{3}$ and DMSO$d_{6}$ were used as solvent for the various compounds to record ${ }^{1} \mathrm{H}$ - and ${ }^{13} \mathrm{C}$-NMR spectra. Molecular ion peaks were recorded as $m / z$, ESI-Mass spectra on a PerkinElmer spectrometer performing at $12.5 \mathrm{eV}$. Carlo Erba EA 1108 CHNS-O automatic analyzer was used for the elemental analysis. 


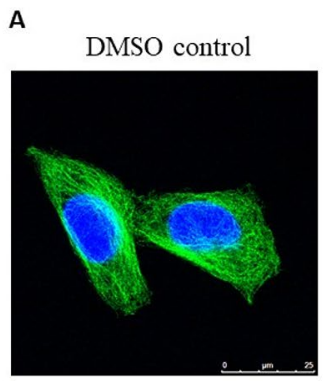

$1 \mu \mathrm{M}$ Vincristine

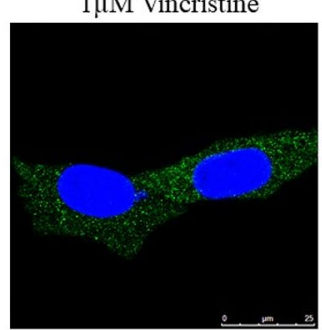

B

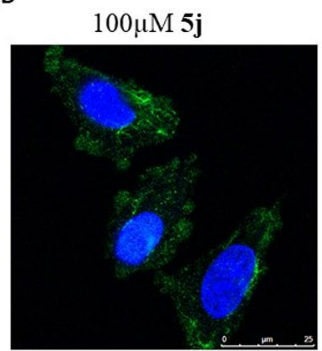

$100 \mu \mathrm{M} 5 \mathrm{q}$

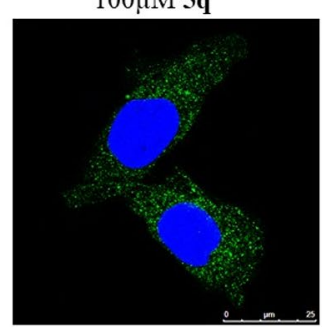

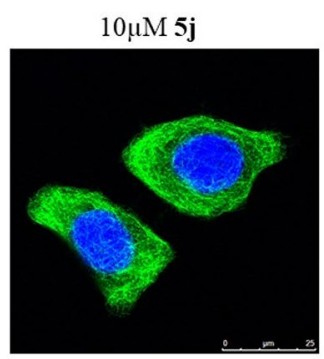

$10 \mu \mathrm{M} 5 \mathbf{q}$

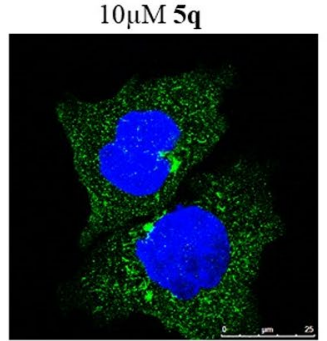

$1 \mu \mathrm{M} 5 \mathbf{q}$

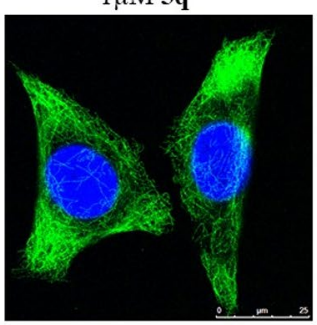

Fig. 5 Immune fluorescence staining of alpha-tubulin in HEp-2 cells: a Representative images of normal alpha-tubulin after treatment with DMSO (top) or typical phenotype after treatment with vincristine (bottom), b Treatment with compounds $( \pm)-5 \mathrm{j}$ and $( \pm)-5 \mathrm{q}$. Green: alpha-tubulin, blue: DAPI. Scale bar: $25 \mu \mathrm{M}$

$6.68(\mathrm{~d}, 1 \mathrm{H}, J=7.6 \mathrm{~Hz}, \mathrm{Ar}-\mathrm{H}), 6.88(d, 1 \mathrm{H}, J=8.0 \mathrm{~Hz}$, $\mathrm{Ar}-\mathrm{H}), 6.94(s, 1 \mathrm{H}, \mathrm{NH}), 6.97(d, 2 \mathrm{H}, J=8.4 \mathrm{~Hz}, \mathrm{Ar}-\mathrm{H})$, $7.11(d, 1 \mathrm{H}, J=8.0 \mathrm{~Hz}, \mathrm{Ar}-\mathrm{H}), 7.94(d, 2 \mathrm{H}, J=8.8 \mathrm{~Hz}$, $\mathrm{Ar}-\mathrm{H}) ;{ }^{13} \mathrm{C}-\mathrm{NMR}\left(100 \mathrm{MHz}, \mathrm{CDCl}_{3}, \delta \mathrm{ppm}\right): 11.7,13.7$, 42.6, 54.9, 55.7, 55.8, 61.0, 107.7, 112.9, 114.3, 119.7, $124.4,127.4,129.4,130.3,130.8,131.2,142.9,146.1$, 151.8, 152.6, 164.6, 193.2; ESI-MS m/z: $507[\mathrm{M}+\mathrm{H}]^{+}$; Analytical calculated formulae $\mathrm{C}_{25} \mathrm{H}_{26} \mathrm{~N}_{6} \mathrm{O}_{4} \mathrm{~S}: \mathrm{C}, 59.27 ; \mathrm{H}$, 5.17; N, 16.59; S, 6.33; Found: C, 59.22; H, 5.22; N, 16.53; S, 6.30 .

\section{( \pm )-(3-(3,5-Dimethyl-1H-pyra-} zol-1-yl)-6-(p-tolyl)-6,7-dihydro-5H-[1,2,4] triazolo[3,4-b][1,3,4] thiadiazin-7-yl)(p-tolyl)methanone (5b)

White solid; yield 91\%; m.p.: 194-196 ${ }^{\circ} \mathrm{C}$; IR (KBr, $\mathrm{v}_{\max } /$ $\left.\mathrm{cm}^{-1}\right)$ : $3135(\mathrm{NH}), 1681(\mathrm{C}=\mathrm{O}) ;{ }^{1} \mathrm{H}-\mathrm{NMR}(400 \mathrm{MHz}$, $\left.\mathrm{CDCl}_{3}, \delta \mathrm{ppm}\right): 2.22\left(s, 3 \mathrm{H}, \mathrm{CH}_{3}\right), 2.30\left(s, 3 \mathrm{H}, \mathrm{CH}_{3}\right), 2.38$ $\left(s, 3 \mathrm{H}, \mathrm{CH}_{3}\right), 2.43\left(s, 3 \mathrm{H}, \mathrm{CH}_{3}\right), 5.05$ (unresolved doublet, $1 \mathrm{H}, \mathrm{CH}), 5.25(d, 1 \mathrm{H}, J=5.2 \mathrm{~Hz}, \mathrm{CH}), 6.00(s, 1 \mathrm{H}, \mathrm{CH}$ of pyrazole ring), $7.11(d, 2 \mathrm{H}, J=8.0 \mathrm{~Hz}, \mathrm{Ar}-\mathrm{H}), 7.29(d, 4 \mathrm{H}$, $J=7.2 \mathrm{~Hz}, \mathrm{Ar}-\mathrm{H}), 7.42(s, 1 \mathrm{H}, \mathrm{NH}), 7.80(d, 2 \mathrm{H}, J=8.0 \mathrm{~Hz}$, $\mathrm{Ar}-\mathrm{H}) ;{ }^{13} \mathrm{C}-\mathrm{NMR}\left(100 \mathrm{MHz}, \mathrm{CDCl}_{3}, \delta \mathrm{ppm}\right): 11.9,13.6$, 21.1, 21.8, 44.2, 59.3, 107.8, 127.3, 128.8, 129.7, 129.8, 132.0, 132.7, 138.8, 141.3, 143.1, 145.6, 145.7, 151.8, 193.7; ESI-MS m/z: $445[\mathrm{M}+\mathrm{H}]^{+}$; Analytical calculated formulae $\mathrm{C}_{24} \mathrm{H}_{24} \mathrm{~N}_{6} \mathrm{OS}$ : C, 64.84; H, 5.44; N, 18.90; S, 7.21; Found: C, 64.89; H, 5.40; N, 18.85; S, 7.18. 
( \pm )-(3-(3,5-Dimethyl-1H-pyra-

zol-1-yl)-6-(4-nitrophenyl)-6,7-dihydro-5H-[1,2,4] triazolo[3,4-b][1,3,4] thiadiazin-7-yl)(4-nitrophenyl) methanone $(5 \mathrm{c})$

Yellow solid; yield 83\%; m.p.: $242-244{ }^{\circ} \mathrm{C}$; IR (KBr, $v_{\max }{ }^{\prime}$ $\left.\mathrm{cm}^{-1}\right)$ : $3302(\mathrm{NH}), 1614(-\mathrm{C}=\mathrm{O}) ;{ }^{1} \mathrm{H}-\mathrm{NMR}(400 \mathrm{MHz}$, $\left.\mathrm{CDCl}_{3}, \delta \mathrm{ppm}\right): 2.16\left(s, 3 \mathrm{H}, \mathrm{CH}_{3}\right), 2.44\left(s, 3 \mathrm{H}, \mathrm{CH}_{3}\right), 5.05$ $(d, 1 \mathrm{H}, J=6.0 \mathrm{~Hz}, \mathrm{CH}), 5.20(d, 1 \mathrm{H}, J=6.0 \mathrm{~Hz}, \mathrm{CH}), 6.00$ $(s, 1 \mathrm{H}, \mathrm{CH}$ of pyrazole ring), $7.30(d, 2 \mathrm{H}, J=8.4 \mathrm{~Hz}, \mathrm{Ar}-\mathrm{H})$, $7.38(d, 2 \mathrm{H}, J=8.4 \mathrm{~Hz}, \mathrm{Ar}-\mathrm{H}), 7.65(d, 2 \mathrm{H}, J=8.1 \mathrm{~Hz}$, $\mathrm{Ar}-\mathrm{H}), 7.73(s, 1 \mathrm{H}, \mathrm{NH}), 7.77(d, 2 \mathrm{H}, J=8.4 \mathrm{~Hz}, \mathrm{Ar}-\mathrm{H})$; ${ }^{13} \mathrm{C}$-NMR $\left(100 \mathrm{MHz}, \mathrm{CDCl}_{3}, \delta \mathrm{ppm}\right): 12.0,13.5,44.1$, 59.1, 107.9, 128.9, 129.0, 129.2, 129.9, 131.9, 134.3, 134.9, 141.0, 143.1, 145.5, 146.0, 151.8, 193.5; ESI-MS m/z: 507 $[\mathrm{M}+\mathrm{H}]^{+}$; Analytical calculated formulae $\mathrm{C}_{22} \mathrm{H}_{18} \mathrm{~N}_{8} \mathrm{O}_{5} \mathrm{~S}: \mathrm{C}$, 52.17; H, 3.58; N, 22.12; S, 6.33; Found: C, 52.23; H, 3.54; N, 22.17; S, 6.30 .

( \pm )-(4-Chlorophenyl)(3-(3,5-dimethyl-1H-pyrazol-1-yl)-6-(3-methoxyphenyl)-6,7-dihydro-5H-[1,2,4]triazolo[3,4-b][1,3,4][1,3,4] thiadiazin-7-yl)methanone (5d)

Cream color solid; yield 86\%; m.p.: $188-190{ }^{\circ} \mathrm{C}$; IR (KBr, $\left.v_{\max } / \mathrm{cm}^{-1}\right)$ : $3138(\mathrm{NH}), 1692(\mathrm{C}=\mathrm{O}) ;{ }^{1} \mathrm{H}-\mathrm{NMR}(400 \mathrm{MHz}$, $\left.\mathrm{CDCl}_{3}, \delta \mathrm{ppm}\right): 2.19\left(s, 3 \mathrm{H}, \mathrm{CH}_{3}\right), 2.41\left(s, 3 \mathrm{H}, \mathrm{CH}_{3}\right), 3.74$ $\left(s, 3 \mathrm{H}, \mathrm{OCH}_{3}\right), 5.04(\mathrm{t}, 1 \mathrm{H}, J=4.0 \mathrm{~Hz}, \mathrm{CH}), 5.25(d, 1 \mathrm{H}$, $J=4.4 \mathrm{~Hz}, \mathrm{CH}), 6.00(s, 1 \mathrm{H}, \mathrm{CH}$ of pyrazole ring), 6.83 $(d, 1 \mathrm{H}, J=6.8 \mathrm{~Hz}, \mathrm{Ar}-\mathrm{H}), 6.96(s, 1 \mathrm{H}, \mathrm{Ar}-\mathrm{H}), 6.98(d, 1 \mathrm{H}$, $J=6.4 \mathrm{~Hz}, \mathrm{Ar}-\mathrm{H}), 7.23(\mathrm{t}, 1 \mathrm{H}, J=6.4 \mathrm{~Hz}, \mathrm{Ar}-\mathrm{H}), 7.47$ $(d, 2 \mathrm{H}, J=6.4 \mathrm{~Hz}, \mathrm{Ar}-\mathrm{H}), 7.58(s, 1 \mathrm{H}, \mathrm{NH}), 7.84(d, 2 \mathrm{H}$, $J=6.8 \mathrm{~Hz}, \mathrm{Ar}-\mathrm{H}) ;{ }^{13} \mathrm{C}-\mathrm{NMR}\left(100 \mathrm{MHz}, \mathrm{CDCl}_{3}, \delta \mathrm{ppm}\right)$ : 12.0, 13.6, 44.9, 55.3, 59.8, 107.9, 113.3, 114.4, 119.4, $129.5,130.1,130.2,132.9,137.1,140.9,141.2,143.2$, 145.5, 151.8, 160.0 192.9; ESI-MS $m / z: 481[\mathrm{M}+\mathrm{H}]^{+}$; Analytical calculated formulae $\mathrm{C}_{23} \mathrm{H}_{21} \mathrm{ClN}_{6} \mathrm{O}_{2} \mathrm{~S}$ : C, 57.44; H, 4.40; N, 17.47; S, 6.67; Found: C, 57.48; H, 4.45; N, 17.42; S, 6.62 .

( \pm )-(6-(2-Bromophenyl)-3-(3,5-dimethyl-1H-pyrazol-1-yl)-6,7-dihydro-5H-[1,2,4]triazolo[3,4-b][1,3,4] thiadiazin-7-yl)(4-fluorophenyl)methanone (5e)

Golden yellow color solid; yield 90\%; m.p.: $195-197{ }^{\circ} \mathrm{C}$; IR (KBr, $\left.v_{\max } / \mathrm{cm}^{-1}\right)$ : $3138(\mathrm{NH}), 1692(\mathrm{C}=\mathrm{O}) ;{ }^{1} \mathrm{H}-\mathrm{NMR}$ (400 MHz, $\left.\mathrm{CDCl}_{3}, \delta \mathrm{ppm}\right): 2.15\left(s, 3 \mathrm{H}, \mathrm{CH}_{3}\right), 2.48(s$, $\left.3 \mathrm{H}, \mathrm{CH}_{3}\right), 5.22(d, 1 \mathrm{H}, J=4.0 \mathrm{~Hz}, \mathrm{CH}), 5.50(\mathrm{t}, 1 \mathrm{H}$, $J=4.8 \mathrm{~Hz}, \mathrm{CH}), 5.99(s, 1 \mathrm{H}, \mathrm{CH}$ of pyrazole ring $), 7.17$ $(s, 1 \mathrm{H}, \mathrm{NH}), 7.20(d, 2 \mathrm{H}, J=8.4 \mathrm{~Hz}, \mathrm{Ar}-\mathrm{H}), 7.24(d, 1 \mathrm{H}$, $J=2.0 \mathrm{~Hz}, \mathrm{Ar}-\mathrm{H}), 7.59(d, 1 \mathrm{H}, J=7.6 \mathrm{~Hz}, \mathrm{Ar}-\mathrm{H}), 7.78(d$, $1 \mathrm{H}, J=5.6 \mathrm{~Hz}, \mathrm{Ar}-\mathrm{H}), 8.01(d \mathrm{~d}, 2 \mathrm{H}, J=8.8 \mathrm{~Hz}, J=5,2 \mathrm{~Hz}$, $\mathrm{Ar}-\mathrm{H}) ;{ }^{13} \mathrm{C}-\mathrm{NMR}\left(100 \mathrm{MHz}, \mathrm{CDCl}_{3}, \delta \mathrm{ppm}\right): 11.9,13.3$,
$43.1,58.3,107.8,116.3,116.5,123.2,128.1,128.6,130.2$, $131.7,131.8,133.4,136.4,140.0,142.9,145.5,151.7$, 166.2, 167.8 192.5; ESI-MS m/z: $515[\mathrm{M}+2]^{+}$; Analytical calculated formulae $\mathrm{C}_{22} \mathrm{H}_{18} \mathrm{BrFN}_{6} \mathrm{OS}: \mathrm{C}, 51.47 ; \mathrm{H}, 3.53 ; \mathrm{N}$, 16.37; S, 6.25; Found: C, 51.42; H, 3.57; N, 16.32; S, 6.20.

\section{( \pm )-(6-(2-Bromophenyl)-3-(3,5-dimethyl-1H-pyra-} zol-1-yl)-6,7-dihydro-5H-[1,2,4]triazolo[3,4-b][1,3,4] thiadiazin-7-yl)(p-tolyl)methanone (5f)

Lemon yellow color solid; yield 92\%; m.p.: 201-203 ${ }^{\circ} \mathrm{C}$; IR ( $\left.\mathrm{KBr}, v_{\max } / \mathrm{cm}^{-1}\right): 3148(\mathrm{NH}), 1673(\mathrm{C}=\mathrm{O}) ;{ }^{1} \mathrm{H}-\mathrm{NMR}$ $\left(400 \mathrm{MHz}, \mathrm{CDCl}_{3}, \delta \mathrm{ppm}\right): 2.20\left(s, 3 \mathrm{H}, \mathrm{CH}_{3}\right), 2.44(s, 3 \mathrm{H}$, $\left.\mathrm{CH}_{3}\right), 2.46\left(s, 3 \mathrm{H}, \mathrm{CH}_{3}\right), 5.22(d, 1 \mathrm{H}, J=4.0 \mathrm{~Hz}, \mathrm{CH}-), 5.49$ (t, $1 \mathrm{H}, J=4.8 \mathrm{~Hz}, \mathrm{CH}), 6.00(s, 1 \mathrm{H}, \mathrm{CH}$ of pyrazole ring), $7.19(\mathrm{t}, 2 \mathrm{H}, J=8.0 \mathrm{~Hz}, \mathrm{Ar}-\mathrm{H}), 7.23(s, 1 \mathrm{H}, \mathrm{NH}), 7.31(d$, $2 \mathrm{H}, J=8.0 \mathrm{~Hz}, \mathrm{Ar}-\mathrm{H}), 7.58(d, 1 \mathrm{H}, J=7.6 \mathrm{~Hz}, \mathrm{Ar}-\mathrm{H}), 7.65$ $(d, 1 \mathrm{H}, J=5.2 \mathrm{~Hz}, \mathrm{Ar}-\mathrm{H}), 7.86(d, 2 \mathrm{H}, J=8.0 \mathrm{~Hz}, \mathrm{Ar}-\mathrm{H})$; ${ }^{13} \mathrm{C}$-NMR (100 MHz, $\left.\mathrm{CDCl}_{3}, \delta \mathrm{ppm}\right): 11.9,13.5,21.8$, $42.9,57.8,107.8,123.0,128.1,128.6,128.9,129.9,130.2$, 131.7, 133.4, 136.6 140.1, 142.9, 145.6, 145.7, 151.8, 193.7; ESI-MS $m / z: 511[\mathrm{M}+2]^{+}$; Analytical calculated formulae $\mathrm{C}_{23} \mathrm{H}_{21} \mathrm{BrN}_{6} \mathrm{OS}$ : C, 54.23; H, 4.16; N, 16.50; S, 6.29; Found: C, 54.28; H, 4.21; N, 16.44; S, 6.33 .

\section{( \pm )-(4-Bromophenyl)}

(6-(4-chlorophenyl)-3-(3,5-dimethyl-1H-pyra-

zol-1-yl)-6,7-dihydro-5H- triazolo[3,4-b][1,3,4] thiadiazin-7-yl)methanone (5g)

White solid; yield 90\%; m.p.: 205-207 ${ }^{\circ} \mathrm{C}$; IR (KBr, $v_{\max }$ ' $\left.\mathrm{cm}^{-1}\right)$ : $3291(\mathrm{NH}), 1688(\mathrm{C}=\mathrm{O}) ;{ }^{1} \mathrm{H}-\mathrm{NMR}(400 \mathrm{MHz}$, $\left.\mathrm{CDCl}_{3}, \delta \mathrm{ppm}\right): 2.16\left(s, 3 \mathrm{H},-\mathrm{CH}_{3}\right), 2.44\left(s, 3 \mathrm{H}, \mathrm{CH}_{3}\right)$, $5.05(d \mathrm{~d}, 1 \mathrm{H}, J=6.0 \mathrm{~Hz}, J=3.6 \mathrm{~Hz}, \mathrm{CH}-), 5.2(d, 1 \mathrm{H}$, $J=6.0 \mathrm{~Hz}, \mathrm{CH}), 6.00(s, 1 \mathrm{H}, \mathrm{CH}$ of pyrazole ring $), 7.30$ $(d, 2 \mathrm{H}, J=8.4 \mathrm{~Hz}, \mathrm{Ar}-\mathrm{H}), 7.38(d, 2 \mathrm{H}, J=8.4 \mathrm{~Hz}, \mathrm{Ar}-\mathrm{H})$, $7.65(d, 2 \mathrm{H}, J=8.1 \mathrm{~Hz}, \mathrm{Ar}-\mathrm{H}), 7.72(s, 1 \mathrm{H}, \mathrm{NH}), 7.77$ $(d, 2 \mathrm{H}, J=8.4 \mathrm{~Hz}, \mathrm{Ar}-\mathrm{H}) ;{ }^{13} \mathrm{C}-\mathrm{NMR}\left(100 \mathrm{MHz}, \mathrm{CDCl}_{3}\right.$, $\delta \mathrm{ppm}): 12.0,13.5,44.9,59.5,107.9,129.0,129.3,132.1$, 130.2 132.6, 133.2, 134.2, 135.1, 140.6, 143.1, 145.3, 151.8, 192.8; ESI-MS m/z: 531 [M+2] ${ }^{+}$; Analytical calculated formulae $\mathrm{C}_{22} \mathrm{H}_{18} \mathrm{BrClN}_{6} \mathrm{OS}: \mathrm{C}, 49.87 ; \mathrm{H}, 3.42 ; \mathrm{N}, 15.86 ; \mathrm{S}$, 6.05; Found: C, 49.84; H, 3.48; N, 15.83; S, 6.12.

( \pm )-(6-(4-Chlorophenyl)-3-(3,5-dimethyl-1H-pyrazol-1-yl)-6,7-dihydro-5H-[1,2,4]triazolo[3,4-b] $[1,3,4]$ thiadiazin-7-yl)(p-tolyl)methanone (5 h)

White solid; yield 93\%; m.p.: 214-216 ${ }^{\circ} \mathrm{C}$; IR (KBr, $v_{\max } /$ $\left.\mathrm{cm}^{-1}\right)$ : $3219(\mathrm{NH}), 1675(\mathrm{C}=\mathrm{O}) ;{ }^{1} \mathrm{H}-\mathrm{NMR}(400 \mathrm{MHz}$, $\mathrm{CDCl}_{3}+$ DMSO- $\left.d_{6}, \delta \mathrm{ppm}\right): 2.25\left(s, 3 \mathrm{H}, \mathrm{CH}_{3}\right), 2.31(s, 3 \mathrm{H}$, $\left.\mathrm{CH}_{3}\right), 2.44\left(s, 3 \mathrm{H}, \mathrm{CH}_{3}\right), 3.21(s, 1 \mathrm{H}, \mathrm{NH}), 4.99$ (unresolved singlet, $1 \mathrm{H}, \mathrm{CH}), 5.69(d, 1 \mathrm{H}, J=5.2 \mathrm{~Hz}, \mathrm{CH}), 6.06(s, 1 \mathrm{H}$, 
$\mathrm{CH}$ of pyrazole ring), $7.19(d, 1 \mathrm{H}, J=7.2 \mathrm{~Hz}, \mathrm{Ar}-\mathrm{H}), 7.28$ $(d, 1 \mathrm{H}, J=6.4 \mathrm{~Hz}, \mathrm{Ar}-\mathrm{H}), 7.33(d, 2 \mathrm{H}, J=7.6 \mathrm{~Hz}, \mathrm{Ar}-\mathrm{H})$, $7.47(s, 2 \mathrm{H}, \mathrm{Ar}-\mathrm{H}), 7.90(s, 2 \mathrm{H}, \mathrm{Ar}-\mathrm{H}),{ }^{13} \mathrm{C}-\mathrm{NMR}(100 \mathrm{MHz}$, $\left.\mathrm{CDCl}_{3}+\mathrm{DMSO}-d_{6}, \delta \mathrm{ppm}\right): 11.4,13.7,21.8,42.5,58.2$, 107.6, 128.9, 129.1, 129.3, 129.8, 132.2, 133.9, 135.2, 141.8, 142.8, 145.6, 146.6, 151.4, 194.3; ESI-MS m/z: $465[\mathrm{M}+\mathrm{H}]^{+}$; Analytical calculated formulae $\mathrm{C}_{23} \mathrm{H}_{21} \mathrm{ClN}_{6} \mathrm{OS}: \mathrm{C}, 59.41 ; \mathrm{H}$, 4.55; N, 18.07; S, 6.90; Found: C, 59.45; H, 4.51; N, 18.10; $\mathrm{S}, 6.85$.

\section{( \pm )-(3-(3,5-Dimethyl-1H-pyra-}

zol-1-yl)-6-(4-hydroxy-3,5-dimethoxyphenyl)-6,7-dihydro-5H- triazolo[3,4-b][1,3,4]thiadiazin-7-yl) (phenyl)methanone (5i)

Green solid; yield 89\%; m.p.: 196-198 ${ }^{\circ} \mathrm{C}$; IR (KBr, $v_{\max }$ ' $\left.\mathrm{cm}^{-1}\right): 3435(\mathrm{OH}), 3134(\mathrm{NH}), 1653(\mathrm{C}=\mathrm{O}) ;{ }^{1} \mathrm{H}-\mathrm{NMR}$ $\left(400 \mathrm{MHz}, \mathrm{CDCl}_{3}+\right.$ DMSO- $\left.d_{6}, \delta \mathrm{ppm}\right): 2.21\left(s, 3 \mathrm{H}, \mathrm{CH}_{3}\right)$, $2.25\left(s, 3 \mathrm{H}, \mathrm{CH}_{3}\right), 3.71\left(s, 6 \mathrm{H}, \mathrm{OCH}_{3}\right), 4.80(\mathrm{t}, 1 \mathrm{H}, J=6.8 \mathrm{~Hz}$, $\mathrm{CH}), 5.86(d, 1 \mathrm{H}, J=6.0 \mathrm{~Hz}, \mathrm{CH}), 6.11(s, 1 \mathrm{H}, \mathrm{CH}$ of pyrazole ring), $6.35(s, 1 \mathrm{H}, \mathrm{OH}), 6.77(s, 2 \mathrm{H}, \mathrm{Ar}-\mathrm{H}), 7.07(s, 1 \mathrm{H}, \mathrm{NH})$, 7.54 (t, $2 \mathrm{H}, J=8.0 \mathrm{~Hz}, \mathrm{Ar}-\mathrm{H}), 7.67$ (t, $1 \mathrm{H}, J=7.2 \mathrm{~Hz}, \mathrm{Ar}-\mathrm{H})$, $8.00(d, 2 \mathrm{H}, J=7.2 \mathrm{~Hz}, \mathrm{Ar}-\mathrm{H}) ;{ }^{13} \mathrm{C}-\mathrm{NMR}\left(100 \mathrm{MHz}, \mathrm{CDCl}_{3}\right.$, $\delta$ ppm): 11.9, 13.5, 45.4, 56.2, 60.8, 104.8, 107.8, 128.7, 129.1, 131.0, 134.5, 134.7, 138.4, 141.5, 143.2, 145.4, 151.7, 153.5, 194.1; ESI-MS $m / z: 493[\mathrm{M}+\mathrm{H}]^{+}$; Analytical calculated formulae $\mathrm{C}_{24} \mathrm{H}_{24} \mathrm{~N}_{6} \mathrm{O}_{4} \mathrm{~S}: \mathrm{C}, 58.52 ; \mathrm{H}, 4.91 ; \mathrm{N}, 17.06 ; \mathrm{S}, 6.51$; Found: C, 58.57; H, 4.94; N, 17.10; S, 6.47.

\section{( \pm )-(3-(3,5-Dimethyl-1H-pyra-}

zol-1-yl)-6-(3,4,5-trifluorophenyl)-6,7-dihydro-5H-[1,2,4]triazole $[3,4-b][1,3,4]$ thiadiazin-7-yl) (p-tolyl)methanone (5j)

White solid; yield 92\%; m.p.: $215-217{ }^{\circ} \mathrm{C}$; IR (KBr, $v_{\max }$ ' $\left.\mathrm{cm}^{-1}\right)$ : $3219(\mathrm{NH}), 1674(\mathrm{C}=\mathrm{O}) ;{ }^{1} \mathrm{H}-\mathrm{NMR}\left(400 \mathrm{MHz}, \mathrm{CDCl}_{3}\right.$, $\delta \mathrm{ppm}): 2.19\left(s, 3 \mathrm{H}, \mathrm{CH}_{3}\right), 2.43\left(s, 3 \mathrm{H}, \mathrm{CH}_{3}\right), 2.50(s, 3 \mathrm{H}$, $\left.\mathrm{CH}_{3}\right), 3.48(s, 1 \mathrm{H}, \mathrm{NH}), 5.035$ (t, $\left.1 \mathrm{H}, J=8.0 \mathrm{~Hz}, \mathrm{CH}\right), 5.48$ (d, $1 \mathrm{H}, J=8.0 \mathrm{~Hz}, \mathrm{CH}), 6.00(s, 1 \mathrm{H}, \mathrm{CH}$ of pyrazole ring), 6.67 (t, $2 \mathrm{H}, J=8.4 \mathrm{~Hz}, \mathrm{Ar}-\mathrm{H}), 7.30(d, 2 \mathrm{H}, J=8.4 \mathrm{~Hz}, \mathrm{Ar}-\mathrm{H}), 7.82(d$, $2 \mathrm{H}, J=8.0 \mathrm{~Hz}, \mathrm{Ar}-\mathrm{H}) ;{ }^{13} \mathrm{C}-\mathrm{NMR}\left(100 \mathrm{MHz}, \mathrm{CDCl}_{3}, \delta \mathrm{ppm}\right)$ : 11.9, 13.5, 21.8, 44.1, 53.1, 101.3, 107.9, 127.5, 128.9, 129.9, 129.8, 131.6, 141.3, 143.2, 145.6, 146.1, 151.8, 160.0, 192.2; ESI-MS $m / z: 485[\mathrm{M}+\mathrm{H}]^{+}$; Analytical calculated formulae $\mathrm{C}_{23} \mathrm{H}_{19} \mathrm{~F}_{3} \mathrm{~N}_{6} \mathrm{OS}$ : C, 57.02; H, 3.95; N, 17.35; S, 6.62; Found: C, 57.17; H, 3.99; N, 17.39; S, 6.62.
( \pm )-(3-(3,5-Dimethyl-1H-pyrazol-1-yl)-6-(3,4,5-trifluorophenyl)-6,7-dihydro-5H-[1,2,4]triazole $[3,4-b][1,3,4]$ thiadiazin-7-yl) (phenyl)methanone (5 k)

Light yellow color solid; yield 90\%; m.p.: $182-184{ }^{\circ} \mathrm{C}$; IR (KBr, $\left.v_{\max } / \mathrm{cm}^{-1}\right): 3207(\mathrm{NH}), 1681(\mathrm{C}=\mathrm{O}) ;{ }^{1} \mathrm{H}-\mathrm{NMR}$ (400 MHz, $\left.\mathrm{CDCl}_{3}, \delta \mathrm{ppm}\right): 2.15\left(s, 3 \mathrm{H}, \mathrm{CH}_{3}\right), 2.49(s$, $\left.3 \mathrm{H}, \mathrm{CH}_{3}\right), 5.34(\mathrm{t}, 1 \mathrm{H}, J=8.0 \mathrm{~Hz}, \mathrm{CH}-), 5.56(d, 1 \mathrm{H}$, $J=8.4 \mathrm{~Hz}, \mathrm{CH}), 5.99(s, 1 \mathrm{H}, \mathrm{CH}$ of pyrazole ring), 6.67 $(\mathrm{t}, 2 \mathrm{H}, J=8.4 \mathrm{~Hz}, \mathrm{Ar}-\mathrm{H}), 7.15(s, 1 \mathrm{H}, \mathrm{NH}), 7.51(\mathrm{t}, 2 \mathrm{H}$, $J=7.6 \mathrm{~Hz}, \mathrm{Ar}-\mathrm{H}), 7.64(\mathrm{t}, 1 \mathrm{H}, J=7.2 \mathrm{~Hz}, \mathrm{Ar}-\mathrm{H}), 7.94$ $(d, 2 \mathrm{H}, J=7.6 \mathrm{~Hz}, \mathrm{Ar}-\mathrm{H}) ;{ }^{13} \mathrm{C}-\mathrm{NMR}\left(100 \mathrm{MHz}, \mathrm{CDCl}_{3}\right.$, $\delta$ ppm): 11.9, 13.4, 44.3, 53.2, 101.3, 107.9, 127.5, 128.9, $129.1,129.2,130.4,134.8,134.9,141.2,143.2,145.6$, 151.8, 192.7; ESI-MS m/z: $471[\mathrm{M}+\mathrm{H}]^{+}$; Analytical calculated formulae $\mathrm{C}_{22} \mathrm{H}_{17} \mathrm{~F}_{3} \mathrm{~N}_{6} \mathrm{OS}$ : C, 56.16; H, 3.64; N, 17.86; S, 6.82; Found: C, 56.12; H, 3.60; N, 17.90; S, 6.87.

\section{( \pm )-(3-(3,5-Dimethyl-1H-pyra-}

zol-1-yl)-6-(3,4,5-trimethoxyphenyl)-6,7-dihydro-5H-triazole[3,4-b][1,3,4]thiadiazin-7-yl)(4-fluorophenyl)methanone (5 I)

White solid; yield 88\%; m.p.: $196-198{ }^{\circ} \mathrm{C}$; IR (KBr, $v_{\max }$ ' $\left.\mathrm{cm}^{-1}\right)$ : $3211(\mathrm{NH}), 1668(\mathrm{C}=\mathrm{O}) ;{ }^{1} \mathrm{H}-\mathrm{NMR}(400 \mathrm{MHz}$, $\mathrm{CDCl}_{3}+$ DMSO- $\left.d_{6}, \delta \mathrm{ppm}\right): 2.18\left(s, 3 \mathrm{H}, \mathrm{CH}_{3}\right), 2.22(s, 3 \mathrm{H}$, $\left.\mathrm{CH}_{3}\right), 3.69\left(s, 3 \mathrm{H}, \mathrm{OCH}_{3}\right), 3.70\left(s, 3 \mathrm{H}, \mathrm{OCH}_{3}\right), 3.87(s, 3 \mathrm{H}$, $\left.\mathrm{OCH}_{3}\right), 4.45(\mathrm{t}, 1 \mathrm{H}, J=6.8 \mathrm{~Hz}, \mathrm{CH}), 5.83(d, 1 \mathrm{H}, J=6.4 \mathrm{~Hz}$, $\mathrm{CH}), 6.15(s, 1 \mathrm{H}, \mathrm{CH}$ of pyrazole ring $), 6.87(s, 1 \mathrm{H}$, $\mathrm{Ar}-\mathrm{H}), 6.97(s, 1 \mathrm{H}, \mathrm{Ar}-\mathrm{H}), 7.07(s, 1 \mathrm{H}, \mathrm{NH}), 7.12(d, 2 \mathrm{H}$, $J=7.2 \mathrm{~Hz}, \mathrm{Ar}-\mathrm{H}), 8.01(d, 2 \mathrm{H}, J=8.4 \mathrm{~Hz}, \mathrm{Ar}-\mathrm{H}) ;{ }^{13} \mathrm{C}-\mathrm{NMR}$ (100 MHz, $\left.\mathrm{CDCl}_{3}, \delta \mathrm{ppm}\right): 11.7,13.7,42.5,54.9,55.7$, 55.8, 61.0, 107.7, 112.9, 114.3, 119.7, 124.4, 127.4, 129.4, 131.2, 142.9, 151.7, 152.6, 164.6, 193.2;ESI-MS m/z: 525 $[\mathrm{M}+\mathrm{H}]^{+}$; Analytical calculated formulae $\mathrm{C}_{25} \mathrm{H}_{25} \mathrm{FN}_{6} \mathrm{O}_{4} \mathrm{~S}: \mathrm{C}$, 57.24; H, 4.80; N, 16.02; S, 6.11; Found: C, 57.20; H, 4.85; $\mathrm{N}, 16.17 ; \mathrm{S}, 6.15$.

\section{( \pm )-(3-(3,5-Dimethyl-1H-pyra-} zol-1-yl)-6-(3,4,5-trimethoxyphenyl)-6,7-dihydro-5H-[1,2,4]triazole [3,4-b][1,3,4]thiadiazin-7-yl) (phenyl)methanone (5m)

Golden yellow color solid; yield 94\%; m.p.: $192-194{ }^{\circ} \mathrm{C}$; IR (KBr, $\left.v_{\max } / \mathrm{cm}^{-1}\right): 3129(\mathrm{NH}), 1680(\mathrm{C}=\mathrm{O}) ;{ }^{1} \mathrm{H}-\mathrm{NMR}$ (400 MHz, $\left.\mathrm{CDCl}_{3}, \delta \mathrm{ppm}\right): 2.19\left(s, 3 \mathrm{H}, \mathrm{CH}_{3}\right), 2.39(s$, $\left.3 \mathrm{H}, \mathrm{CH}_{3}\right), 3.48\left(s, 6 \mathrm{H}, \mathrm{OCH}_{3}\right), 3.78\left(s, 3 \mathrm{H}, \mathrm{OCH}_{3}\right), 5.00$ $(\mathrm{t}, 1 \mathrm{H}, J=5.2 \mathrm{~Hz}, \mathrm{CH}), 5.31(d, 1 \mathrm{H}, J=5.6 \mathrm{~Hz}, \mathrm{CH}), 6.00$ $(s, 1 \mathrm{H}, \mathrm{CH}$ of pyrazole ring), $6.66(s, 2 \mathrm{H}, \mathrm{Ar}-\mathrm{H}), 7.50$ $(\mathrm{t}, 2 \mathrm{H}, J=7.6 \mathrm{~Hz}, \mathrm{Ar}-\mathrm{H}), 7.59(s, 1 \mathrm{H}, \mathrm{NH}), 7.63(\mathrm{t}, 1 \mathrm{H}$, $J=7.6 \mathrm{~Hz}, \mathrm{Ar}-\mathrm{H}), 7.90(d, 2 \mathrm{H}, J=7.6 \mathrm{~Hz}, \mathrm{Ar}-\mathrm{H}) ;{ }^{13} \mathrm{C}-\mathrm{NMR}$ $\left(100 \mathrm{MHz}, \mathrm{CDCl}_{3}, \delta \mathrm{ppm}\right): 11.9,13.5,45.4,56.2,60.6$, 
60.8, 104.8, 107.8, 128.7, 129.1, 131.0, 134.5, 134.7, 138.4, 141.5, 143.2, 145.4, 151.7, 153.5, 194.1; ESI-MS m/z: 507 $[\mathrm{M}+\mathrm{H}]^{+}$; Analytical calculated formulae $\mathrm{C}_{25} \mathrm{H}_{26} \mathrm{~N}_{6} \mathrm{O}_{4} \mathrm{~S}: \mathrm{C}$, 59.27; H, 5.17; N, 16.59; S, 6.33; Found: C, 59.24; H, 5.20; N, 16.54; S, 6.38.

\section{( \pm )-(6-(2-Chlorophenyl)-3-(3,5-dimethyl-1H-pyra-} zol-1-yl)-6,7-dihydro-5H-[1,2,4]triazolo[3,4-b][1,3,4] thiadiazin-7-yl)(4-methoxyphenyl)methanone (5n)

White solid; yield $86 \%$; m.p.: $198-200{ }^{\circ} \mathrm{C}$; IR $\left(\mathrm{KBr}, \mathrm{v}_{\max }{ }^{\prime}\right.$ $\left.\mathrm{cm}^{-1}\right)$ : $3143(\mathrm{NH}), 1671(\mathrm{C}=\mathrm{O})$; ${ }^{1} \mathrm{H}-\mathrm{NMR}(400 \mathrm{MHz}$, $\left.\mathrm{CDCl}_{3}, \delta \mathrm{ppm}\right): 2.21\left(s, 3 \mathrm{H}, \mathrm{CH}_{3}\right), 2.45\left(s, 3 \mathrm{H}, \mathrm{CH}_{3}\right), 3.90$ $\left(s, 3 \mathrm{H}, \mathrm{OCH}_{3}\right), 5.18(d, 1 \mathrm{H}, J=4.0 \mathrm{~Hz}, \mathrm{CH}), 5.50(\mathrm{t}, 1 \mathrm{H}$, $J=4.4 \mathrm{~Hz}, \mathrm{CH}), 6.00(s, 1 \mathrm{H}, \mathrm{CH}$ of pyrazole ring $), 6.98$ $(d, 2 \mathrm{H}, J=8.8 \mathrm{~Hz}, \mathrm{Ar}-\mathrm{H}), 7.16-7.23(\mathrm{~m}, 2 \mathrm{H}, \mathrm{Ar}-\mathrm{H}), 7.29$ $(s, 1 \mathrm{H}, \mathrm{NH}), 7.40(d, 1 \mathrm{H}, J=8.0 \mathrm{~Hz}, \mathrm{Ar}-\mathrm{H}), 7.60(d, 1 \mathrm{H}$, $J=4.8 \mathrm{~Hz}, \mathrm{Ar}-\mathrm{H}), 7.94(d, 2 \mathrm{H}, J=8.4 \mathrm{~Hz}, \mathrm{Ar}-\mathrm{H}) ;{ }^{13} \mathrm{C}-$ NMR (100 MHz, $\left.\mathrm{CDCl}_{3}, \delta \mathrm{ppm}\right): 12.0,13.5,21.8,44.1$, $59.1,107.9,128.9,129.0,129.2,129.9,131.9,134.3,134.9$, 141.0, 143.1, 145.5, 145.9, 151.8, 193.5; ESI-MS m/z: 481 $[\mathrm{M}+\mathrm{H}]^{+}$; Analytical calculated formulae $\mathrm{C}_{23} \mathrm{H}_{21} \mathrm{ClN}_{6} \mathrm{O}_{2} \mathrm{~S}$ : C, 57.44; H, 4.40; N, 17.47; S, 6.67; Found: C, 57.40; H, $4.45 ; \mathrm{N}, 17.1 ; \mathrm{S}, 6.62$.

( \pm )-(6-(2,4-Dimethoxyphenyl)-3-(3,5-dimethyl-1H-pyrazol-1-yl)-6,7-dihydro-5H-[1,2,4]triazole [3,4-b][1,3,4]thiadiazin-7-yl)(4-methoxyphenyl) methanone (50)

Yellow solid; yield 94\%; m.p.: $150-152{ }^{\circ} \mathrm{C}$; IR (KBr, $v_{\max }{ }^{\prime}$ $\left.\mathrm{cm}^{-1}\right)$ : $3129(\mathrm{NH}), 1680(\mathrm{C}=\mathrm{O})$; ${ }^{1} \mathrm{H}-\mathrm{NMR}(400 \mathrm{MHz}$, DMSO- $d_{6}, \delta$ ppm): $2.19\left(s, 3 \mathrm{H}, \mathrm{CH}_{3}\right), 2.22\left(s, 3 \mathrm{H}, \mathrm{CH}_{3}\right)$, $3.69\left(s, 3 \mathrm{H}, \mathrm{OCH}_{3}\right), 3.70\left(s, 3 \mathrm{H}, \mathrm{OCH}_{3}\right), 3.87\left(s, 3 \mathrm{H}, \mathrm{OCH}_{3}\right)$, $4.45(\mathrm{t}, 1 \mathrm{H}, J=6.8 \mathrm{~Hz}, \mathrm{CH}), 5.83(d, 1 \mathrm{H}, J=6.4 \mathrm{~Hz}, \mathrm{CH})$, $6.16(s, 1 \mathrm{H}, \mathrm{CH}$ of pyrazole ring), $6.86(d, 1 \mathrm{H}, J=8.4 \mathrm{~Hz}$, $\mathrm{Ar}-\mathrm{H}), 6.98(d, 1 \mathrm{H}, J=8.4 \mathrm{~Hz}, \mathrm{Ar}-\mathrm{H}), 7.07(s, 1 \mathrm{H}, \mathrm{NH})$, $7.10(d, 2 \mathrm{H}, J=8.0 \mathrm{~Hz}, \mathrm{Ar}-\mathrm{H}), 7.12(s, 1 \mathrm{H}, \mathrm{Ar}-\mathrm{H}), 8.01$ $(d, 2 \mathrm{H}, J=8.4 \mathrm{~Hz}, \mathrm{Ar}-\mathrm{H}) ;{ }^{13} \mathrm{C}-\mathrm{NMR}\left(100 \mathrm{MHz}, \mathrm{CDCl}_{3}, \delta\right.$ ppm): 11.7, 13.7, 42.6, 54.9, 55.7, 55.8, 61.0, 107.7, 112.9, $114.3,119.7,124.4,127.4,129.4,130.3,130.8,131.2$, 142.9, 146.1, 151.8, 152.6, 164.6, 193.2; ESI-MS m/z: 507 $[\mathrm{M}+\mathrm{H}]^{+}$; Analytical calculated formulae $\mathrm{C}_{25} \mathrm{H}_{26} \mathrm{~N}_{6} \mathrm{O}_{4} \mathrm{~S}: \mathrm{C}$, 59.27; H, 5.17; N, 16.59; S, 6.33; Found: C, 59.24; H, 5.14; N, 16.63; S, 6.30 .

( \pm )-(6-(2,4-dimethoxyphenyl)-3-(3,5-dimethyl-1H-pyrazol-1-yl)-5H-[1,2,4]triazolo[3,4-b][1,3,4] thiadiazin-7-yl)(4-nitrophenyl)methanone (5p)

Yellow solid; yield $88 \%$; m.p.: $236-238^{\circ} \mathrm{C}$; IR (KBr, $v_{\max }{ }^{\prime}$ $\left.\mathrm{cm}^{-1}\right): 3135(\mathrm{NH}), 1681(\mathrm{C}=\mathrm{O}) ;{ }^{1} \mathrm{H}-\mathrm{NMR}(400 \mathrm{MHz}$, $\left.\mathrm{CDCl}_{3}, \delta \mathrm{ppm}\right): 2.35\left(s, 3 \mathrm{H}, \mathrm{CH}_{3}\right), 2.42\left(s, 3 \mathrm{H}, \mathrm{CH}_{3}\right)$, $3.79\left(s, 3 \mathrm{H}, \mathrm{OCH}_{3}\right), 3.88\left(s, 3 \mathrm{H}, \mathrm{OCH}_{3}\right), 6.08(s, 1 \mathrm{H}$, $\mathrm{CH}$ of pyrazole ring $), 6.48(s, 1 \mathrm{H}, \mathrm{Ar}-\mathrm{H}), 6.62(d \mathrm{~d}, 1 \mathrm{H}$, $J=8.4 \mathrm{~Hz}, J=6.4 \mathrm{~Hz}, \mathrm{Ar}-\mathrm{H}), 7.27(s, 1 \mathrm{H}, \mathrm{NH}), 7.43(d$, $1 \mathrm{H}, J=8.8 \mathrm{~Hz}, \mathrm{Ar}-\mathrm{H}), 7.95(d, 2 \mathrm{H}, J=8.8 \mathrm{~Hz}, \mathrm{Ar}-\mathrm{H}), 8.31$ $(d, 2 \mathrm{H}, J=8.8 \mathrm{~Hz}, \mathrm{Ar}-\mathrm{H}) ;{ }^{13} \mathrm{C}-\mathrm{NMR}\left(100 \mathrm{MHz}, \mathrm{CDCl}_{3}\right.$, $\delta \mathrm{ppm}): 11.7,13.7,55.6,55.7,98.5,104.7,108.1,112.9$, $114.6,123.9,131.0,131.2,138.3,141.2,143.6,146.5$, 149.4, 152.4, 16.2, 159.1, 163.3, 196.0; Analytical calculated formulae $\mathrm{C}_{24} \mathrm{H}_{21} \mathrm{~N}_{7} \mathrm{O}_{5} \mathrm{~S}: \mathrm{C}, 55.48 ; \mathrm{H}, 4.07 ; \mathrm{N}, 18.87$; S, 6.17; Found: C, 55.43; H, 4.02; N, 18.92; S, 6.21.

\section{( \pm )-(3-(3,5-Dimethyl-1H-pyra-}

zol-1-yl)-6-(furan-2-yl)-6,7-dihydro-5H-[1,2,4] triazolo[3,4-b][1,3,4] thiadiazin-7-yl)(4-methoxyphenyl)methanone (5q)

Brown solid; yield 87\%; m.p.: $152-154{ }^{\circ} \mathrm{C}$; IR (KBr, $v_{\max }$ ' $\left.\mathrm{cm}^{-1}\right)$ : $3143(\mathrm{NH}), 1671(\mathrm{C}=\mathrm{O})$; ${ }^{1} \mathrm{H}-\mathrm{NMR}$ (400 MHz, $\left.\mathrm{CDCl}_{3}, \delta \mathrm{ppm}\right): 2.30\left(s, 3 \mathrm{H}, \mathrm{CH}_{3}\right), 2.34\left(s, 3 \mathrm{H}, \mathrm{CH}_{3}\right), 3.92$ $\left(s, 3 \mathrm{H}, \mathrm{OCH}_{3}\right), 5.23$ (unresolved singlet, $2 \mathrm{H}, \mathrm{CH}$ and $\mathrm{CH}$ ), $6.03(s, 1 \mathrm{H}, \mathrm{CH}$ of pyrazole ring $), 6.30(s, 1 \mathrm{H}, \mathrm{CH}-), 6.32(s$, $1 \mathrm{H}, \mathrm{CH}), 7.02(d, 3 \mathrm{H}, J=8.8 \mathrm{~Hz}, \mathrm{Ar}-\mathrm{H}), 7.35(s, 1 \mathrm{H}, \mathrm{NH})$, $7.94(d, 2 \mathrm{H}, J=8.4 \mathrm{~Hz}, \mathrm{Ar}-\mathrm{H})$; ESI-MS $m / z 437[\mathrm{M}+\mathrm{H}]^{+}$; Analytical calculated formulae $\mathrm{C}_{21} \mathrm{H}_{20} \mathrm{~N}_{6} \mathrm{O}_{3} \mathrm{~S}: \mathrm{C}, 57.79 ; \mathrm{H}$, 4.62; N, 19.25; S, 7.35; Found: C, 57.83; H, 4.66; N, 19.21; S, 7.31.

( \pm )-(3-(3,5-Dimethyl-1H-pyra-

zol-1-yl)-6-(furan-2-yl)-6,7-dihydro-5H-[1,2,4] triazolo[3,4-b] $[1,3,4]$ thiadiazin-7-yl)(p-tolyl)methanone $(5 r)$

Brown solid; yield $85 \%$; m.p.: $149-151^{\circ} \mathrm{C}$; IR (KBr, $v_{\max }$ ' $\left.\mathrm{cm}^{-1}\right)$ : $3204(\mathrm{NH}), 1666(\mathrm{C}=\mathrm{O}) ;{ }^{1} \mathrm{H}-\mathrm{NMR}(400 \mathrm{MHz}$, $\left.\mathrm{CDCl}_{3}, \delta \mathrm{ppm}\right): 2.30\left(s, 3 \mathrm{H}, \mathrm{CH}_{3}\right), 2.34\left(s, 3 \mathrm{H}, \mathrm{CH}_{3}\right), 2.46(s$, $\left.3 \mathrm{H}, \mathrm{CH}_{3}\right), 5.26$ (unresolved singlet, $\left.2 \mathrm{H}, \mathrm{CH}-\right), 6.03(s, 1 \mathrm{H}$, $\mathrm{CH}$ of pyrazole ring), $6.31(d, 2 \mathrm{H}, J=8.0 \mathrm{~Hz}, \mathrm{Ar}-\mathrm{H}), 7.03$ $(s, 1 \mathrm{H}, \mathrm{NH}), 7.35(d, 3 \mathrm{H}, J=8.8 \mathrm{~Hz}, \mathrm{Ar}-\mathrm{H}), 7.85(d, 2 \mathrm{H}$, $J=8.0 \mathrm{~Hz}, \mathrm{Ar}-\mathrm{H}) ;{ }^{13} \mathrm{C}-\mathrm{NMR}\left(100 \mathrm{MHz}, \mathrm{CDCl}_{3}, \delta \mathrm{ppm}\right)$ : $11.6,13.7,21.9,39.8,53.0,107.7,109.2,111.0,128.9$, 130.0, 131.6, 140.6, 142.7, 143.1, 146.1, 146.7, 148.3, 152.0, 194.4; ESI-MS m/z: $421[\mathrm{M}+\mathrm{H}]^{+}$; Analytical calculated formulae $\mathrm{C}_{21} \mathrm{H}_{20} \mathrm{~N}_{6} \mathrm{O}_{2} \mathrm{~S}: \mathrm{C}, 59.98 ; \mathrm{H}, 4.79 ; \mathrm{N}, 19.99$; S, 7.63; Found: C, 59.94; H, 4.7; N, 19.94; S, 7.68.

( \pm )-(3-(3,5-Dimethyl-1H-pyrazol-1-yl)-6-(thiophen-2-yl)-6,7-dihydro-5H-[1,2,4] triazolo[3,4-b][1,3,4] thiadiazin-7-yl)(4-nitrophenyl) methanone (5s)

Golden color solid; yield 89\%; m.p.: $204-206^{\circ} \mathrm{C}$; IR (KBr, $\left.v_{\max } / \mathrm{cm}^{-1}\right)$ : 3281(NH), $1696(\mathrm{C}=\mathrm{O}) ;{ }^{1} \mathrm{H}-\mathrm{NMR}(400 \mathrm{MHz}$, $\left.\mathrm{CDCl}_{3}+\mathrm{DMSO}-d_{6}, \delta \mathrm{ppm}\right): 2.26\left(s, 3 \mathrm{H}, \mathrm{CH}_{3}\right), 2.27(s, 3 \mathrm{H}$, 
$\left.\mathrm{CH}_{3}\right), 5.47(\mathrm{t}, 1 \mathrm{H}, J=3.6 \mathrm{~Hz}, \mathrm{CH}), 5.88(d, 1 \mathrm{H}, J=3.2 \mathrm{~Hz}$, $\mathrm{CH}), 6.10(s, 1 \mathrm{H}, \mathrm{CH}$ of pyrazole ring $), 6.97(\mathrm{t}, 1 \mathrm{H}$, $J=4.4 \mathrm{~Hz}, \mathrm{Ar}-\mathrm{H}), 7.17(d, 1 \mathrm{H}, J=4.4 \mathrm{~Hz}, \mathrm{Ar}-\mathrm{H}), 7.31(d$, $1 \mathrm{H}, J=4.8 \mathrm{~Hz}, \mathrm{Ar}-\mathrm{H}), 7.35$ (s, 1H, NH), 8.37 (s, 4H, Ar-H); ${ }^{13} \mathrm{C}-\mathrm{NMR}\left(100 \mathrm{MHz}, \mathrm{CDCl}_{3}+\right.$ DMSO- $\left.d_{6}, \delta \mathrm{ppm}\right): 11.4$, $13.8,42.9,53.4,107.5,124.1,126.3,126.5,127.4,129.3$, $130.8,139.9,142.9,146.9,149.6,150.7,151.3,194.5$; ESI-MS $m / z: 468[\mathrm{M}+\mathrm{H}]^{+}$; Analytical calculated formulae $\mathrm{C}_{20} \mathrm{H}_{17} \mathrm{~N}_{7} \mathrm{O}_{3} \mathrm{~S}$ : C, 51.38; H, 3.67; N, 20.97; S, 13.72; Found: C, 51.34; H, 3.62; N, 20.94; S, 13.76.

\section{( \pm )-(3-(3,5-Dimethyl-1H-pyra- zol-1-yl)-6-(furan-2-yl)-6,7-dihydro-5H-[1,2,4] triazolo[3,4-b][1,3,4] thiadiazin-7-yl)(4-nitrophenyl) methanone (5t)}

White solid; yield $92 \%$; m.p.: $201-20{ }^{\circ} \mathrm{C}$; IR $\left(\mathrm{KBr}, \mathrm{v}_{\max }{ }^{\prime}\right.$ $\left.\mathrm{cm}^{-1}\right)$ : $3278(\mathrm{NH}), 1698(\mathrm{C}=\mathrm{O}) ;{ }^{1} \mathrm{H}-\mathrm{NMR}(400 \mathrm{MHz}$, $\left.\mathrm{CDCl}_{3}, \delta \mathrm{ppm}\right): 2.25\left(s, 3 \mathrm{H}, \mathrm{CH}_{3}\right), 2.27\left(s, 3 \mathrm{H}, \mathrm{CH}_{3}\right)$, 5.28-5.32 (m, 1H, CH), $5.78(d, 1 \mathrm{H}, J=4.0 \mathrm{~Hz}, \mathrm{CH}), 6.08$ $(s, 1 \mathrm{H}, \mathrm{CH}$ of pyrazole ring $), 6.45(s, 1 \mathrm{H}, \mathrm{NH}), 7.14(d, 1 \mathrm{H}$, $J=4.0 \mathrm{~Hz}, \mathrm{Ar}-\mathrm{H}), 7.48(d, 1 \mathrm{H}, J=4.0 \mathrm{~Hz}, \mathrm{Ar}-\mathrm{H}), 7.85(\mathrm{t}$, $1 \mathrm{H}, J=8.4 \mathrm{~Hz}, \mathrm{Ar}-\mathrm{H}), 8.29$ (d, 2H $J=8.4 \mathrm{~Hz}, \mathrm{Ar}-\mathrm{H}), 8.33$ $(d, 2 \mathrm{H}, J=7.2 \mathrm{~Hz}, \mathrm{Ar}-\mathrm{H}) ;{ }^{13} \mathrm{C}-\mathrm{NMR}\left(100 \mathrm{MHz}, \mathrm{CDCl}_{3}\right.$, $\delta$ ppm): 11.2, 13.8, 49.8, 52.5, 107.5, 109.2, 111.0, 124.1, $124.3,130.4,130.6,142.9,143.0,148.1,149.1,150.7151 .3$, 194.0; ESI-MS $\mathrm{m} / z: 452[\mathrm{M}+\mathrm{H}]^{+}$; Analytical calculated formulae $\mathrm{C}_{20} \mathrm{H}_{17} \mathrm{~N}_{7} \mathrm{O}_{4} \mathrm{~S}: \mathrm{C}, 53.21 ; \mathrm{H}, 3.80 ; \mathrm{N}, 21.72 ; \mathrm{S}, 7.10$; Found: C, 53.25; H, 3.85; N, 21.7; S, 7.15.

\section{X-ray crystallography}

The diffraction data was collected on Bruker APEX2 single crystal X-ray diffractometere quipped with a CCD area detector system, graphite mono chromator and a Mo- $\mathrm{K}_{\alpha}$ fine focus sealed tube $(\lambda=0.71073 \AA)$. Bruker SAINT PLUS was used for data reduction, SHELXT-2014 [49] was used for structure solution and SHELXL-2018 [50] was used for full-matrix least-squares refinement. Mercury 3.3 [51] was used for molecular graphics. All non-hydrogen atoms were refined using anisotropic thermal parameters. All hydrogen atoms bound to carbons were positioned geometrically and refined using a riding model. Important crystallographic data and table for bond distances and bond angles were provided in supporting information.

Supplementary Information The online version contains supplementary material available at https://doi.org/10.1007/s11030-021-10258-8.

Acknowledgements We are thankful to the Head, Department of Chemistry and Director, National Institute of Technology, Warangal, Telangana, for providing infrastructure facilities and one of the authors P.C. Jilloju is thankful to MHRD government of India, for providing research fellowship.

\section{References}

1. Wan J-P, Cao S, Liu Y (2016) Base-promoted synthesis of N-substituted 1,2,3-triazoles via enaminone-azide cycloaddition involving regitz diazo transfer. Org Lett 18:6034-6037. https://doi.org/ 10.1021/acs.orglett.6b02975

2. Wang X-Y, Zhong Y-F, Mo Z-Y et al (2021) Synthesis of seleno oxindoles via electrochemical cyclization of $\mathrm{N}$-arylacrylamides with diorganyl diselenides. Adv Synth Catal 363:208-214. https:// doi.org/10.1002/adsc.202001192

3. Zhong P-F, Lin H-M, Wang L-W et al (2020) Electrochemically enabled synthesis of sulfide imidazopyridines via a radical cyclization cascade. Green Chem 22:6334-6339. https://doi.org/10. 1039/D0GC02125C

4. Guo Y, Wang G, Wei L, Wan J-P (2019) Domino C-H sulfonylation and pyrazole annulation for fully substituted pyrazole synthesis in water using hydrophilic enaminones. J Org Chem 84:2984-2990. https://doi.org/10.1021/acs.joc.8b02897

5. Wang M-R, Deng L, Liu G-C et al (2019) Porous organic polymerderived nanopalladium catalysts for chemoselective synthesis of antitumor benzofuro[2,3- b ]pyrazine from 2-bromophenol and isonitriles. Org Lett 21:4929-4932. https://doi.org/10.1021/acs. orglett.9b01230

6. Wan J-P, Jing Y, Hu C, Sheng S (2016) Metal-free synthesis of fully substituted pyridines via ring construction based on the domino reactions of enaminones and aldehydes. J Org Chem 81:6826-6831. https://doi.org/10.1021/acs.joc.6b01149

7. Tong W, Li W-H, He Y et al (2018) Palladium-metalated porous organic polymers as recyclable catalysts for the chemioselective synthesis of thiazoles from thiobenzamides and isonitriles. Org Lett 20:2494-2498. https://doi.org/10.1021/acs.orglett.8b00886

8. Kaur P, Chawla A (2017) 1,2,4-Triazole: a review of pharmacological activities. Int Res J Pharm 8:10-29. https://doi.org/10. 7897/2230-8407.087112

9. Bayrak H, Demirbas A, Demirbas N, Karaoglu SA (2009) Synthesis of some new 1,2,4-triazoles starting from isonicotinic acid hydrazide and evaluation of their antimicrobial activities. Eur J Med Chem 44:4362-4366. https://doi.org/10.1016/j.ejmech.2009. 05.022

10. Burman WJ (2010) Rip van winkle wakes up: Development of tuberculosis treatment in the 21st century. Clin Infect Dis 50:165172. https://doi.org/10.1086/651487

11. Abdel-Aal MT, El-Sayed WA, El-Kosy SM, El-Ashry ESH (2008) Synthesis and antiviral evaluation of novel 5-(N-arylaminomethyl-1,3,4- oxadiazol-2-yl)hydrazines and their sugars, 1,2,4-triazoles, tetrazoles and pyrazolyl derivatives. Arch Pharm (Weinheim) 341:307-313. https://doi.org/10.1002/ardp.200700154

12. Benci K, Suhina T, Mandić L et al (2011) Novel 1,2,4-triazole and purine acyclic cyclopropane nucleoside analogues: synthesis, antiviral and cytostatic activity evaluations. Antivir Chem Chemother 21:221-230. https://doi.org/10.3851/IMP1762

13. Rezaei Z, Khabnadideh S, Pakshir K et al (2009) Design, synthesis, and antifungal activity of triazole and benzotriazole derivatives. Eur J Med Chem. https://doi.org/10.1016/j.ejmech.2008.07. 012

14. Andrews B, Ahmed M (2014) Synthesis and characterization of pyrimidine bearing 1,2,4-triazole derivatives and their potential antifungal action. Int J ChemTech Res 6:1013-1021

15. Mathew V, Keshavayya J, Vaidya VP (2006) Heterocyclic system containing bridgehead nitrogen atom: synthesis and pharmacological activities of some substituted 1,2,4-triazolo[3,4-b]-1,3,4thiadiazoles. Eur J Med Chem 41:1048-1058. https://doi.org/10. 1016/j.ejmech.2006.03.018

16. Jiang B, Huang X, Yao H et al (2014) Discovery of potential anti-inflammatory drugs: Diaryl-1,2,4-triazoles bearing 
$\mathrm{N}$-hydroxyurea moiety as dual inhibitors of cyclooxygenase-2 and 5-lipoxygenase. Org Biomol Chem 12:2114-2127. https:// doi.org/10.1039/c3ob41936c

17. Abuo-Rahma GEDAA, Abdel-Aziz M, Beshr EAM, Ali TFS (2014) 1,2,4-Triazole/oxime hybrids as new strategy for nitric oxide donors: synthesis, anti-inflammatory, ulceroginicity and antiproliferative activities. Eur J Med Chem 71:185-198. https://doi.org/10.1016/j.ejmech.2013.11.006

18. Shivarama Holla B, Veerendra B, Shivananda M, Poojary B (2003) Synthesis characterization and anticancer activity studies on some Mannich bases derived from 1,2,4-triazoles. Eur J Med Chem 38:759-767. https://doi.org/10.1016/S0223-5234(03) 00128-4

19. Plech T, Luszczki JJ, Wujec M et al (2013) Synthesis, characterization and preliminary anticonvulsant evaluation of some 4-alkyl-1,2,4-triazoles. Eur J Med Chem 60:208-215. https:// doi.org/10.1016/j.ejmech.2012.11.026

20. Cetin A, Geçibesler IH (2015) Evaluation as antioxidant agents of 1,2,4-triazole derivatives: effects of essential functional groups. J Appl Pharm Sci 5:120-126. https://doi.org/10.7324/ JAPS.2015.50620

21. Ongini E, Monopoli A, Cacciari B, Giovanni Baraldi P (2001) Selective adenosine A2A receptor antagonists. Farm 56:87-90. https://doi.org/10.1016/S0014-827X(01)01024-2

22. Messore A, Corona A, Madia VN et al (2020) Pyrrolyl pyrazoles as non-diketo acid inhibitors of the HIV-1 ribonuclease $\mathrm{H}$ function of reverse transcriptase. ACS Med Chem Lett 11:798-805. https://doi.org/10.1021/acsmedchemlett.9b00617

23. Kim J, Lee D, Park C et al (2012) Discovery of phenylaminopyridine derivatives as novel HIV-1 non-nucleoside reverse transcriptase inhibitors. ACS Med Chem Lett 3:678-682. https:// doi.org/10.1021/ml300146q

24. Jiang B, Fan W, Sun MY et al (2014) Domino reaction of arylglyoxals with pyrazol-5-amines: selective access to pyrazolofused 1,7-naphthyridines, 1,3-diazocanes, and pyrroles. J Org Chem 79:5258-5268. https://doi.org/10.1021/jo500823z

25. Renuka N, Ajay Kumar K (2013) Synthesis and biological evaluation of novel formyl-pyrazoles bearing coumarin moiety as potent antimicrobial and antioxidant agents. Bioorganic Med Chem Lett 23:6406-6409. https://doi.org/10.1016/j.bmcl.2013. 09.053

26. Tewari AK, Srivastava P, Singh VP et al (2010) Novel antiinflammatory agents based on pyrazole based dimeric compounds; design, synthesis, docking and in vivo activity. Chem Pharm Bull (Tokyo) 58:634-638. https://doi.org/10.1248/cpb.58.634

27. Vijesh AM, Isloor AM, Shetty P et al (2013) New pyrazole derivatives containing 1,2,4-triazoles and benzoxazoles as potent antimicrobial and analgesic agents. Eur J Med Chem 62:410-415. https://doi.org/10.1016/j.ejmech.2012.12.057

28. Poce G, Consalvi S, Venditti G et al (2019) Novel pyrazole-containing compounds active against mycobacterium tuberculosis. ACS Med Chem Lett 10:1423-1429. https://doi.org/10.1021/ acsmedchemlett.9b00204

29. Park HJ, Lee K, Park SJ et al (2005) Identification of antitumor activity of pyrazole oxime ethers. Bioorganic Med Chem Lett 15:3307-3312. https://doi.org/10.1016/j.bmcl.2005.03.082

30. Sener A, Kasimogullari R, Sener MK, Genc H (2004) Studies on reactions of cyclic oxalyl compounds with hydrazines or hydrazones. 2. Synthesis and reactions of 4-benzoyl-1-(4-nitrophenyl)5-phenyl- 1H-pyrazole-3-carboxylic acid. Chem Heterocycl Compd 40:1039-1046. https://doi.org/10.1023/B:COHC.00000 46695.00178 .79

31. Menozzi G, Mosti L, Fossa P et al (1997) $\omega$-Dialkylaminoalkyl ethers of phenyl-(5-substituted 1-phenyl-1 H -pyrazol-4-yl)methanols with analgesic and anti-inflammatory activity. J Heterocycl Chem 34:963-968. https://doi.org/10.1002/jhet.5570340339
32. Magedov IV, Manpadi M, Van Slambrouck S et al (2007) Discovery and investigation of antiproliferative and apoptosis-inducing properties of new heterocyclic podophyllotoxin analogues accessible by a one-step multicomponent synthesis. J Med Chem 50:5183-5192. https://doi.org/10.1021/jm070528f

33. Mowbray CE, Braillard S, Speed W et al (2015) Novel aminopyrazole ureas with potent in vitro and in vivo antileishmanial activity. J Med Chem 58:9615-9624. https://doi.org/10.1021/acs. jmedchem.5b01456

34. Zhang B, Li Y-H, Liu Y et al (2015) Design, synthesis and biological evaluation of novel 1,2,4-triazolo [3,4-b][1,3,4] thiadiazines bearing furan and thiophene nucleus. Eur J Med Chem 103:335-342. https://doi.org/10.1016/j.ejmech.2015.08.053

35. Shah TA, Ahmad Z, Mir NA et al (2015) One step synthesis of highly functionalized thiazolo[3\{,\}2-b][1\{,\}2\{,\}4]triazole $\{$, triazolo[1\{,\}5-a]pyrimidine and triazolo[3\{,\}4-b][1\{,\}3\{,\}4] thiadiazine. RSC Adv 5:107931-107937. https://doi.org/10.1039/ C5RA21270G

36. Khan I, Ibrar A, Abbas N (2013) Triazolothiadiazoles and triazolothiadiazines - biologically attractive scaffolds. Eur J Med Chem 63:854-868. https://doi.org/10.1016/j.ejmech.2013.01.060

37. Sumangala V, Poojary B, Chidananda N et al (2012) Facile synthesis, cytotoxic and antimicrobial activity studies of a new group of 6-aryl-3-[4-(methylsulfonyl)benzyl]-7H-[1,2,4]triazolo[3,4-b] [1,3,4]thiadiazines. Eur J Med Chem 54:59-64. https://doi.org/10. 1016/j.ejmech.2012.04.024

38. Khan I, Zaib S, Ibrar A et al (2014) Synthesis, crystal structure and biological evaluation of some novel 1,2,4-triazolo[3,4b]-1,3,4-thiadiazoles and 1,2,4-triazolo[3,4-b]-1,3,4-thiadiazines. Eur J Med Chem 78:167-177. https://doi.org/10.1016/j.ejmech. 2014.03.046

39. Pundeer R, Kiran V, Prakash R et al (2012) $\alpha$, $\alpha$-Dibromoacetophenones mediated synthesis of some new $7 \mathrm{H}-7$ alkoxy-3-alkyl/phenyl-6-aryl-s-triazolo[3,4-b][1,3,4]thiadiazines and their antimicrobial evaluation. Med Chem Res 21:4043-4052. https://doi.org/10.1007/s00044-011-9945-1

40. Saha A, Payra S, Banerjee S (2015) One-pot multicomponent synthesis of highly functionalized bio-active pyrano[2,3-c]pyrazole and benzylpyrazolyl coumarin derivatives using $\mathrm{ZrO} 2$ nanoparticles as a reusable catalyst. Green Chem 17:2859-2866. https:// doi.org/10.1039/C4GC02420F

41. Bhunia A, Porwal D, Gonnade RG, Biju AT (2013) Multicomponent reactions involving arynes, quinolines, and aldehydes. Org Lett 15:4620-4623. https://doi.org/10.1021/ol4023134

42. Cioc RC, Ruijter E, Orru RVA (2014) Multicomponent reactions: advanced tools for sustainable organic synthesis. Green Chem 16:2958-2975. https://doi.org/10.1039/C4GC00013G

43. Jilloju PC, Shyam P, Sanjeev A, Vedula RR (2021) Four-component, one-pot synthesis of (E)-N-benzylidene-3-(benzylthio)5-(3,5-dimethyl-1H-pyrazol-1-yl)-4H-1,2,4-triazol-4-amines and their DNA binding and molecular docking studies. J Mol Struct 1225:129140. https://doi.org/10.1016/j.molstruc.2020.129140

44. Mamidala S, Peddi SR, Aravilli RK et al (2021) Microwave irradiated one pot, three component synthesis of a new series of hybrid coumarin based thiazoles: Antibacterial evaluation and molecular docking studies. J Mol Struct. https://doi.org/10.1016/j.molstruc. 2020.129114

45. Sujatha K, Vedula RR (2019) Multicomponent Efficient synthesis of new $[1,2,4]$ Triazolo[3,4]thiadiazines. J Heterocycl Chem 56:832-838. https://doi.org/10.1002/jhet.3458

46. Jilloju PC, Vedula RR (2018) A facile one-pot three-component synthesis of benzylideneamino-3,5-dimethyl-1H-pyrazoles. Synth Commun 48:1739-1746. https://doi.org/10.1080/00397911.2018. 1458242

47. Div MC, College SC (1989) 4-Amino-3-pyrazolo-1,2,4-triazoles as antimicrobial agents+. Arch Pharm 66:63-66 
48. Al-Etaibi A, John E, Ibrahim MR et al (2011) Stereoselective synthesis of dihydrothiadiazinoazines and dihydrothiadiazinoazoles and their pyrolytic desulfurization ring contraction. Tetrahedron 67:6259-6274. https://doi.org/10.1016/j.tet.2011.06.034

49. Sheldrick GM (2015) SHELXT - Integrated space-group and crystal-structure determination. Acta Crystallogr Sect A Found Crystallogr 71:3-8. https://doi.org/10.1107/S2053273314026370

50. Sheldrick GM (2015) Crystal structure refinement with SHELXL. Acta Crystallogr Sect C Struct Chem 71:3-8. https://doi.org/10. $1107 /$ S2053229614024218
51. Macrae CF, Bruno IJ, Chisholm JA et al (2008) Mercury CSD 2.0 - New features for the visualization and investigation of crystal structures. J Appl Crystallogr 41:466-470. https://doi.org/10. 1107/S0021889807067908

Publisher's Note Springer Nature remains neutral with regard to jurisdictional claims in published maps and institutional affiliations.

\section{Authors and Affiliations}

\section{Parameshwara Chary Jilloju ${ }^{1}$. Leentje Persoons ${ }^{2} \cdot$ Sathish Kumar Kurapati ${ }^{3,4}$ - Dominique Schols ${ }^{2}$. Steven De Jonghe ${ }^{2} \cdot$ Dirk Daelemans $^{2} \cdot$ Rajeswar Rao Vedula $^{1}$ (i)}

\section{Rajeswar Rao Vedula}

rajeswarnitw@gmail.com; vrajesw@nitw.ac.in

1 Department of Chemistry, National Institute of Technology, Warangal, Telangana 506004, India

Department of Microbiology, Immunology and Transplantation, Laboratory of Virology and Chemotherapy, KU Leuven, Rega Institute for Medical Research, Herestraat 49, Leuven, Belgium
3 Department of Chemistry, National Institute of Technology, Andhra Pradesh 534101, India

4 Department of Chemistry, Chaithanya Bharati Institute of Technology, Hyderabad, Telangana 500075, India 